\title{
The troposphere-to-stratosphere transition in kinetic energy spectra and nonlinear spectral fluxes as seen in ECMWF analyses
}

Article

Published Version

Burgess, B. H., Erler, A. R. and Shepherd, T. G. (2013) The troposphere-to-stratosphere transition in kinetic energy spectra and nonlinear spectral fluxes as seen in ECMWF analyses. Journal of the Atmospheric Sciences, 70 (2). pp. 669-687. ISSN 1520-0469 doi: https://doi.org/10.1175/JAS-D-12-0129.1 Available at https://centaur.reading.ac.uk/32106/

It is advisable to refer to the publisher's version if you intend to cite from the work. See Guidance on citing.

Published version at: http://dx.doi.org/10.1175/JAS-D-12-0129.1

To link to this article DOI: http://dx.doi.org/10.1175/JAS-D-12-0129.1

Publisher: American Meteorological Society

All outputs in CentAUR are protected by Intellectual Property Rights law, including copyright law. Copyright and IPR is retained by the creators or other copyright holders. Terms and conditions for use of this material are defined in the End User Agreement. 


\section{CentAUR}

Central Archive at the University of Reading

Reading's research outputs online 


\title{
The Troposphere-to-Stratosphere Transition in Kinetic Energy Spectra and Nonlinear Spectral Fluxes as Seen in ECMWF Analyses
}

\author{
B. H. BuRgesS AND ANDRE R. ERLER \\ Department of Physics, University of Toronto, Toronto, Ontario, Canada \\ THEODORE G. SHEPHERD \\ Department of Physics, University of Toronto, Toronto, Ontario, Canada, and Department of Meteorology, \\ University of Reading, Reading, United Kingdom
}

(Manuscript received 26 April 2012, in final form 25 August 2012)

\begin{abstract}
Global horizontal wavenumber kinetic energy spectra and spectral fluxes of rotational kinetic energy and enstrophy are computed for a range of vertical levels using a T799 ECMWF operational analysis. Above $250 \mathrm{hPa}$, the kinetic energy spectra exhibit a distinct break between steep and shallow spectral ranges, reminiscent of dual power-law spectra seen in aircraft data and high-resolution general circulation models. The break separates a large-scale "balanced" regime in which rotational flow strongly dominates divergent flow and a mesoscale "unbalanced" regime where divergent energy is comparable to or larger than rotational energy. Between 230 and $100 \mathrm{hPa}$, the spectral break shifts to larger scales (from $n=60$ to $n=20$, where $n$ is spherical harmonic index) as the balanced component of the flow preferentially decays. The location of the break remains fairly stable throughout the stratosphere. The spectral break in the analysis occurs at somewhat larger scales than the break seen in aircraft data. Nonlinear spectral fluxes defined for the rotational component of the flow maximize between about 300 and $200 \mathrm{hPa}$. Large-scale turbulence thus centers on the extratropical tropopause region, within which there are two distinct mechanisms of upscale energy transfer: eddy-eddy interactions sourcing the transient energy peak in synoptic scales, and zonal mean-eddy interactions forcing the zonal flow. A well-defined downscale enstrophy flux is clearly evident at these altitudes. In the stratosphere, the transient energy peak moves to planetary scales and zonal mean-eddy interactions become dominant.
\end{abstract}

\section{Introduction}

Analyzing the nonlinear dynamics of the atmospheric circulation within the framework of two-dimensional turbulence theory is of long-standing interest. Although the atmosphere is baroclinic, Charney (1971) argued that quasigeostrophic dynamics leads to quasi-twodimensional behavior at synoptic and larger scales. Within this framework, baroclinic instability provides energy for barotropic motions, and nonlinear interactions associated with the advection term transfer kinetic energy upscale and enstrophy downscale (Salmon 1980). As a result, atmospheric kinetic energy is strongly confined to the largest scales of motion.

Corresponding author address: B. H. Burgess, Department of Physics, University of Toronto, 60 St. George St., Toronto, ON M5S 1A7, Canada.

E-mail: belhburgess@physics.utoronto.ca
Diagnostic studies have largely confirmed this picture. Using the first available global analysis, at T32 spectral resolution (i.e., maximum spherical harmonic index $n=32$, corresponding approximately to a horizontal wavelength of $1200 \mathrm{~km}$ ), Boer and Shepherd (1983) found upscale kinetic energy transfer from synoptic scales to planetary scales and downscale enstrophy transfer from synoptic scales to the smallest resolved scales. Associated with the downscale enstrophy transfer, the upper-tropospheric $(200 \mathrm{hPa})$ kinetic energy spectrum had a power-law scaling over the range $n=10-25$ of approximately $n^{-3}$, in accord with predictions for an enstrophy-cascading two-dimensional turbulent inertial subrange (Kraichnan 1967). At larger scales, the power-law scaling expected for the inverse energy cascade was not observed; Boer and Shepherd attributed this to the stationary component's dominance at planetary scales. 
Trenberth and Solomon (1993) found that the approximate $n^{-3}$ power-law scaling in the upper troposphere extended to $n=70$, the apparent effective resolution limit of their T106 analysis, as evidenced by rapid power drop off at smaller scales. Straus and Ditlevsen (1999) examined troposphere-averaged kinetic energy and enstrophy spectral fluxes in another T106 analysis, and also found kinetic energy and enstrophy fluxes to large and small scales, respectively. Their results differed quantitatively from Boer and Shepherd (1983) in several respects, which is to be expected, since energy and enstrophy transfers into or out of a given scale through nonlinear interactions involve wavenumber triad interactions with, in principle, all other scales. Hence any finite-resolution analysis introduces some truncation error in these quantities, even at the largest scales. In particular, Straus and Ditlevsen (1999) found overwhelmingly upscale kinetic energy flux, with only about $10 \%$ directed downscale. This confirmed, as argued by Boer and Shepherd (1983), that the rather large downscale transfer ( $\sim 25 \%$ of the total) found in their study was an artifact of limited spatial resolution. However, $10 \%$ is still nonnegligible, which leaves open whether the T106 analysis fully captured the upscale energy transfer. Moreover, Straus and Ditlevsen noted that although enstrophy transfer was almost entirely downscale, there was no evidence for an inertial range with constant flux. Instead they found that the enstrophy flux grew with wavenumber until limited by the dissipation range. This implies the downscale enstrophy flux was not fully resolved in their analysis.

It thus remains an open question whether there is a well-defined downscale enstrophy flux in the atmosphere, as posited by two-dimensional turbulence theory, and what spatial resolution is needed to fully capture the upscale energy flux. An equivalent question, in the scenario where baroclinic instability sources twodimensional turbulence, is whether the baroclinic excitation range is spectrally confined. This has implications for required resolution in climate models. If baroclinic energy injection occurs over a limited range of wavenumbers, at sufficient resolution one would expect an enstrophy inertial range with constant spectral flux and resolved upscale energy transfers.

The extent to which two-dimensional turbulence theory applies to the stratosphere, where kinetic energy is primarily injected by upward-propagating planetary waves rather than baroclinic instability, is also of interest. Trenberth and Solomon (1993) examined the 10-hPa level in their study and found no evidence of power-law scaling, but noted that this was the upper level of their analysis. Both questions - the extent to which kinetic energy injection is spectrally confined and can therefore be resolved, and how spectral dynamics differ between the troposphere and stratosphere-motivate reexamining kinetic energy spectra and nonlinear spectral transfers of kinetic energy and enstrophy using a modern meteorological analysis with much higher spatial resolution and a well-resolved stratosphere.

Another reason to perform such a study is to compare with spectra from aircraft data. Nastrom and Gage (1985) showed that the -3 power-law scaling of the upper-tropospheric synoptic-scale kinetic energy spectrum gives way to a much shallower mesoscale spectrum, with slope close to $-5 / 3$ for wavelengths smaller than $400 \mathrm{~km}$ (approximately $n=100$ ). Since its discovery, the origin of this $-5 / 3$ spectrum has been controversial. Standard similarity arguments (Kraichnan 1967) suggest an energy inertial range, but the flux could be in either direction. Certainly, at mesoscales the shallow spectrum cannot represent the three-dimensional Kolmogorov inertial range. Some (e.g., Vallis et al. 1997) have argued, following Lilly (1983), that a quasitwo-dimensional balanced inertial range carrying energy upscale from small-scale mesoscale instabilities such as thunderstorms generates the shallow mesoscale spectrum. Others (e.g., Waite and Bartello 2004 and Lindborg 2006) have argued that it represents a downscale unbalanced energy inertial range, a hypothesis supported by structure-function analysis of the aircraft data (Cho and Lindborg 2001). Bacmeister et al. (1996) also observed a $-5 / 3$ kinetic energy spectrum in research aircraft measurements around 20-km altitude (approximately $50 \mathrm{hPa}$ ), over wavelengths ranging from 150 to $600 \mathrm{~km}(n=60$ 300). At these altitudes, the only conceivable mechanism is upward-propagating internal gravity waves, which is consistent with the "unbalanced" hypothesis for the mesoscale Nastrom-Gage spectrum in the upper troposphere.

A simple way to distinguish between balanced and unbalanced motion is by comparing the relative magnitudes of divergent and rotational flow. For balanced dynamics, whether the small Rossby number balance relevant to synoptic scales or the small Froude number balance relevant to the mesoscale, the divergent motion is much weaker than the rotational motion (Saujani and Shepherd 2006). In contrast, for unbalanced dynamics, the divergent component is comparable to or larger than the rotational component. Notably, while Trenberth and Solomon (1993) found no evidence for a mesoscale regime, which is hardly surprising given the spatial resolution of their analysis, they did note that the divergent kinetic energy spectrum followed a shallow (approximately -2) power law between $n=10$ and the onset of the dissipation range at $n=70$. If the total and divergent energies had maintained the same power laws beyond $n=70$, they would have met (at $200 \mathrm{hPa}$ ) near $n=100$, 
roughly consistent with the Nastrom-Gage spectrum. The much-higher-resolution analysis we study here resolves the mesoscale better, allowing us to probe the region where the divergent and rotational energy spectra would have intersected.

Our purpose is thus to examine horizontal wavenumber spectra of rotational and divergent kinetic energy, as well as nonlinear spectral fluxes of rotational kinetic energy and enstrophy using a modern highresolution operational analysis with a well-resolved stratosphere. In so doing we recognize that meteorological analyses do not necessarily represent the state of the atmosphere, only our best estimate of that state, and that aspects of the analysis not well constrained by measurements will be largely determined by the forecast model. That does not, however, make these aspects uninteresting, since the forecast model encodes our best understanding of the equations governing atmospheric behavior.

We use spherical harmonic decompositions and compute global spectra, noting that tropopause height varies with latitude-a potential limitation of our approach. However, computing global spectra provides continuity with earlier studies (Boer and Shepherd 1983; Trenberth and Solomon 1993; Straus and Ditlevsen 1999), including Nastrom and Gage (1985), whose spectra include both tropical and midlatitude measurements.

For the spectra, the key question is whether the analysis resolves a spectral break consistent with aircraft observations, and how the divergent and rotational components of kinetic energy reflect this. For the spectral fluxes, the key questions are whether the analysis resolves the baroclinic source region and associated downscale enstrophy flux, and how spectral dynamics differ in the stratosphere. Within the troposphere, we focus on the upper troposphere, where the aircraft data are taken, and where the nonlinear spectral fluxes maximize (Boer and Shepherd 1983), consistent with our understanding of synopticscale Rossby wave breaking and eddy momentum fluxes (Held and Hoskins 1985; Randel and Held 1991).

The remainder of the paper is organized as follows. Section 2 describes the European Centre for MediumRange Weather Forecasts (ECMWF) International Polar Year (IPY) data, spherical harmonic decompositions, and changepoint analysis. Kinetic energy spectra are presented in section 3, nonlinear interaction terms and spectral fluxes in section 4 , and in section 5 we summarize and discuss our findings.

\section{Data and methods}

\section{a. Dataset}

The data analyzed herein are horizontal wind fields from the ECMWF "International Polar Year" analysis, included in the Stratospheric Processes and Their Role in Climate-International Polar Year (SPARC-IPY) data archive (Pendlebury 2009), obtainable from the SPARC Data Center at http://www.sparc.sunysb.edu/. The winds are available at $0000,0600,1200$, and 1800 UTC on 91 hybrid model levels. The grid is equally spaced, with resolution $0.25^{\circ} \times 0.25^{\circ}$, resolving total spherical harmonic wavenumber $n=721$. The ECMWF system at the time was T799 on a linear, reduced Gaussian grid, so the IPY data is T799 data interpolated to a regular latitude-longitude grid. To check that interpolation does not significantly affect the spectral slopes at synoptic and mesoscales, we recomputed kinetic energy spectra using the uninterpolated T799 dataset, and found the results virtually unchanged - the IPY and T799 spectra almost coincide well into the dissipation range.

Previous studies (Trenberth and Solomon 1993; Straus and Ditlevsen 1999) have shown that 1 month of global data sufficiently characterizes spectral properties at synoptic and smaller scales, so we consider only January 2008. Planetary-scale spectral properties depend on season; these variations have been explored in previous studies (Boer and Shepherd 1983; Trenberth and Solomon 1993; Straus and Ditlevsen 1999) and are robust over datasets.

\section{b. Spherical harmonic decompositions}

Spherical harmonic decompositions and syntheses were computed from the winds at all available times in the month of January 2008 using the Spherepack 3.2 package from the National Center for Atmospheric Research (NCAR) (Adams and Swarztrauber 1997). We calculate all quantities on model levels, with no interpolation to pressure levels in the lower troposphere, where the pressure varies appreciably over the level. However, our focus is on the upper troposphere and above, where model levels essentially coincide with pressure levels. We have checked that spectra calculated on model and pressure levels coincide at both 250 and $100 \mathrm{hPa}$. Calculating quantities on model levels where possible is preferable since the winds are not interpolated in the vertical.

Relevant scalar fields are expanded in spherical harmonic basis functions $Y_{n}^{m}$ (Boer 1983). The streamfunction expansion, truncated at total spherical harmonic wavenumber $N$, is

$$
\psi(\lambda, \phi)=\sum_{n=0}^{N} \sum_{m=-n}^{n} \psi_{n}^{m} Y_{n}^{m}(\lambda, \phi) .
$$

Here $n$ is the total spherical harmonic wavenumber, $m$ is the zonal wavenumber, and $\psi_{n}^{m}$ are the spherical harmonic coefficients of the streamfunction. The basis 
functions are $Y_{n}^{m}=P_{n, m} e^{i m \lambda}$, where $P_{n, m}$ are the Legendre polynomials. Divergence is expanded similarly.

For spherical harmonics, the transformed Laplacian becomes $-n(n+1) / a^{2}$, where $a$ is Earth's radius. Approximating $n(n+1)$ by $n^{2}$, we obtain

$$
\lambda=\frac{2 \pi a}{n} \simeq \frac{40000 \mathrm{~km}}{n}
$$

so that $n=40$ corresponds to $\lambda=1000 \mathrm{~km}$, for example.

\section{1) SPECTRA}

The total kinetic energy spectrum is (Koshyk and Hamilton 2001)

$$
E_{n}=\frac{1}{4} \frac{a^{2}}{n(n+1)} \sum_{m=-n}^{n}\left(\overline{\left|\zeta_{n}^{m}\right|^{2}}+\overline{\left|\delta_{n}^{m}\right|^{2}}\right),
$$

where the overbar represents a monthly average, and $\delta_{n}^{m}$ and $\zeta_{n}^{m}$ are the spherical harmonic coefficients of divergence and vorticity, respectively. See the appendix for decompositions into rotational and divergent, stationary and transient, and zonal and meridional components. The enstrophy spectrum is

$$
G_{n}=\frac{n(n+1)}{a^{2}} E_{n}^{\mathrm{rot}},
$$

where $E_{n}^{\text {rot }}$ is the rotational kinetic energy.

2) NONLINEAR INTERACTION TERMS AND FLUXES

Starting from the vorticity equation

$$
\frac{\partial \zeta}{\partial t}=-(\mathbf{v} \cdot \nabla) \zeta-D
$$

where $\mathbf{v}=(u, v)$ is rotational velocity, $\zeta$ vorticity, and $D$ divergent effects, sources, and sinks, we calculate nonlinear interaction terms. The spectral space enstrophy equation is

$$
\frac{\partial G_{n}}{\partial t}=J_{n}+D_{n}^{G}
$$

and the kinetic energy equation is

$$
\frac{\partial E_{n}^{\mathrm{rot}}}{\partial t}=I_{n}+D_{n}^{E}
$$

where $J_{n}$ and $I_{n}$ are interaction terms, representing nonlinear transfers of enstrophy and kinetic energy into wavenumber $n$, and $D_{n}^{G}$ and $D_{n}^{E}$ include divergent effects, sources, and sinks of enstrophy and kinetic energy, respectively.

The enstrophy interaction term is given by

$$
J_{n}=-\frac{1}{4} \sum_{m=-n}^{n}\left[\zeta_{n}^{m^{*}}(\mathbf{v} \cdot \nabla \zeta)_{n}^{m}+\zeta_{n}^{m}(\mathbf{v} \cdot \nabla \zeta)_{n}^{m^{*}}\right]
$$

where the asterisk indicates the complex conjugate, and the kinetic energy interaction term is (Boer and Shepherd 1983)

$$
I_{n}=\frac{a^{2}}{n(n+1)} J_{n}
$$

To obtain $J_{n}$ from (8), $\zeta$ and $\mathbf{v} \cdot \nabla \zeta$ were computed at each grid point for each time step using the Spherepack 3.2 subroutines, and then decomposed into spherical harmonics.

Since $I_{n}$ and $J_{n}$ only redistribute kinetic energy and enstrophy among wavenumbers,

$$
\sum_{n=0}^{N} I_{n}=0=\sum_{n=0}^{N} J_{n}
$$

On a finite grid, the flux of kinetic energy is

$$
F_{n+1}=-\sum_{l=0}^{n} I_{l}
$$

and the enstrophy flux is

$$
H_{n+1}=-\sum_{l=0}^{n} J_{l} \text {. }
$$

Equation (10) implies the fluxes vanish at sufficiently large $n$.

In a turbulent inertial range, the fluxes are independent of $n$, the kinetic energy and enstrophy in each wavenumber are time independent, and the interaction terms vanish. Hence, one can determine whether a powerlaw regime might correspond to a true inertial range by checking whether the fluxes are constant with $n$ and the interaction terms vanish.

\section{c. Changepoint analysis}

Changepoint analysis can be used to statistically estimate the location of a regime transition (Hinkley 1969; Toms and Lesperance 2003). The technique consists of fitting to the data linear segments under the constraint that they connect, forming a piecewise differentiable curve. The slope changes discontinuously at the changepoint(s), where the segments meet. The location of the changepoint is not known in advance, but rather calculated as part of the regression.

We use changepoint analysis to identify spectral breaks in the ECMWF kinetic energy spectra and fit slopes to the adjoined scaling regimes. The simplest model, in which two straight lines are joined sharply at the changepoint, gives very good fits to the ECMWF data. The model is 

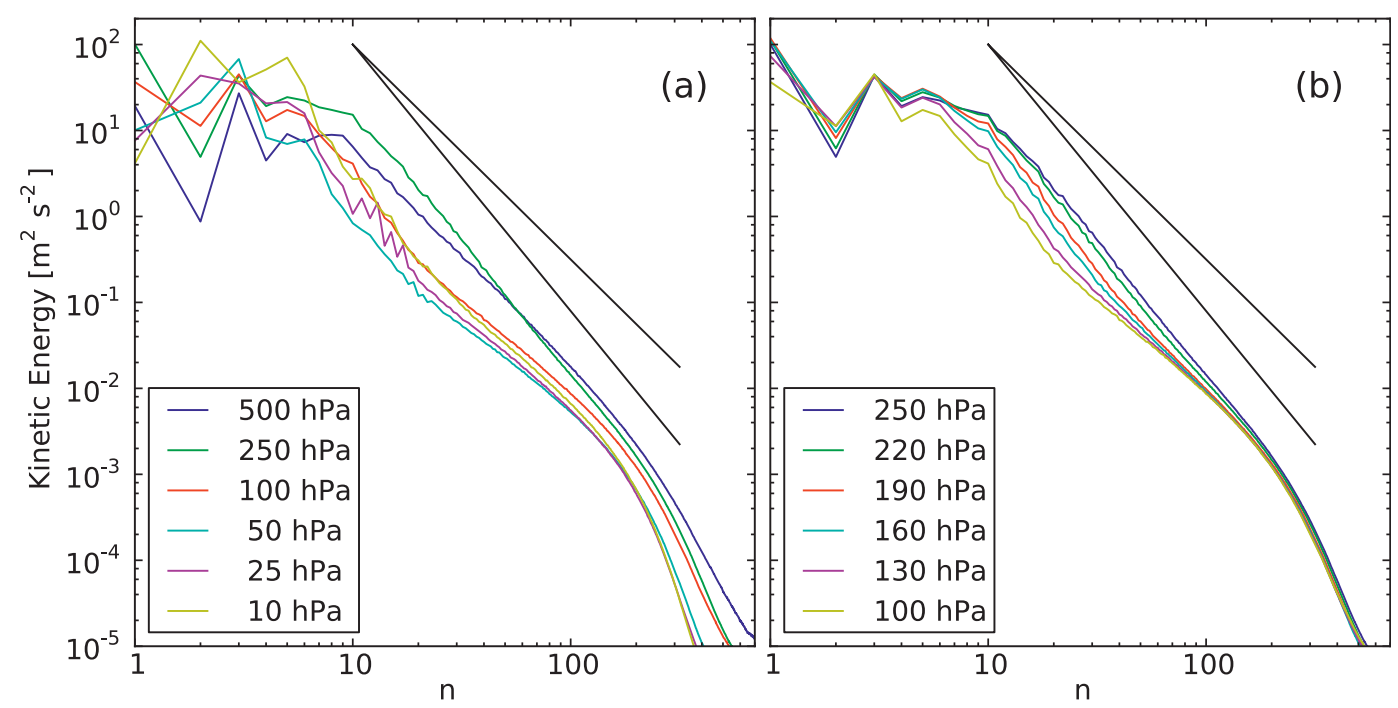

FIG. 1. Kinetic energy spectra at selected model levels (a) spanning the troposphere to stratosphere and (b) in the tropopause region. Lines with slopes of -3.1 and -2.5 have been added to both panels for comparison.

$\ln \left(E_{n}\right)=\left\{\begin{array}{lll}\beta_{0}+\beta_{2} \alpha+\beta_{1} \ln (n)+e_{n}, & \text { for } & \ln (n) \leq \alpha, \\ \beta_{0}+\beta_{1} \alpha+\beta_{2} \ln (n)+e_{n}, & \text { for } & \ln (n)>\alpha,\end{array}\right.$

where $E_{n}$ is the kinetic energy in wavenumber $n$ and $\alpha$ is the natural logarithm of the changepoint wavenumber, $n_{\mathrm{cp}}=\exp (\alpha)$. The parameters $\beta_{1}$ and $\beta_{2}$ are the slopes of the first and second segment. The parameter $\beta_{0}$ is a constant normalization factor without physical significance; $e_{n}$ represents an independent, additive error term with vanishing mean (Toms and Lesperance 2003).

Equation (13) defines a nonlinear statistical model with four free parameters $\left(\alpha, \beta_{0}, \beta_{1}, \beta_{2}\right)$, which must be estimated simultaneously. We used the SciPy scientific Python "leastsq" routine, which relies on the LevenbergMarquardt algorithm (Levenberg 1944) to minimize the root-mean-square error of the model (13) against the data (the kinetic energy spectra). The $95 \%$ confidence interval for each parameter was estimated from the covariance matrix.

The changepoint analysis was performed over the range $n=10-100$ in order to avoid fitting the dissipation range and the peak at planetary scales. Note that the density of data points increases at higher wavenumbers. The following values were used as initial guesses: $\alpha=\ln (20), \beta_{0}=\ln (5400), \beta_{1}=-3.3$, and $\beta_{2}=$ -2.0 ; the results are not sensitive to this particular choice.

\section{Kinetic energy spectra}

Figure 1a shows kinetic energy spectra for levels spanning the midtroposphere $(500 \mathrm{hPa})$ to the midstratosphere $(10 \mathrm{hPa})$. At 500 and $250 \mathrm{hPa}$, a single spectral range extends from about $n=10-100$, with pronounced steepening at higher wavenumbers suggestive of dissipative effects. We define the dissipation range as beginning where the spectra visibly turn over and steepen; $n=100$ is a conservative estimate. The approximate $n^{-3}$ power-law behavior at $250 \mathrm{hPa}$ confirms earlier results (Boer and Shepherd 1983; Trenberth and Solomon 1993) using much-lower-resolution analyses. In the ECMWF analysis, this power law, characteristic of the two-dimensional turbulent enstrophy-cascading subrange, extends into the mesoscale to spatial wavelengths of $400 \mathrm{~km}$ or less. As will be seen in section 4, the $250-\mathrm{hPa}$ level corresponds to the maximum nonlinear spectral fluxes and hence the most intense largescale turbulence. The shallower power law at altitudes below $250 \mathrm{hPa}$ is similar to the behavior seen in Boer and Shepherd (1983).

Spectral amplitudes decrease with altitude immediately above $100 \mathrm{hPa}$ but then start to increase again (due to decreasing density). At $100 \mathrm{hPa}$ and above, the kinetic energy spectra exhibit a spectral break around $n=20$, with a distinctly shallower spectrum and welldefined power law from about $n=20-100$. Note that $n=$ 20 corresponds to a spatial wavelength of about $2000 \mathrm{~km}$. This spectral break between an approximately $n^{-3}$ largescale spectrum and a shallower mesoscale spectrum is reminiscent of the Nastrom-Gage spectrum (Nastrom and Gage 1985). The transition between the distinct behavior at 250 and $100 \mathrm{hPa}$ develops gradually. The changepoint algorithm first detects a spectral break at $226 \mathrm{hPa}$, but it only becomes clearly visible above about $200 \mathrm{hPa}$. The transition is examined in Fig. 1b: the break emerges because of a large decrease in synoptic-scale 


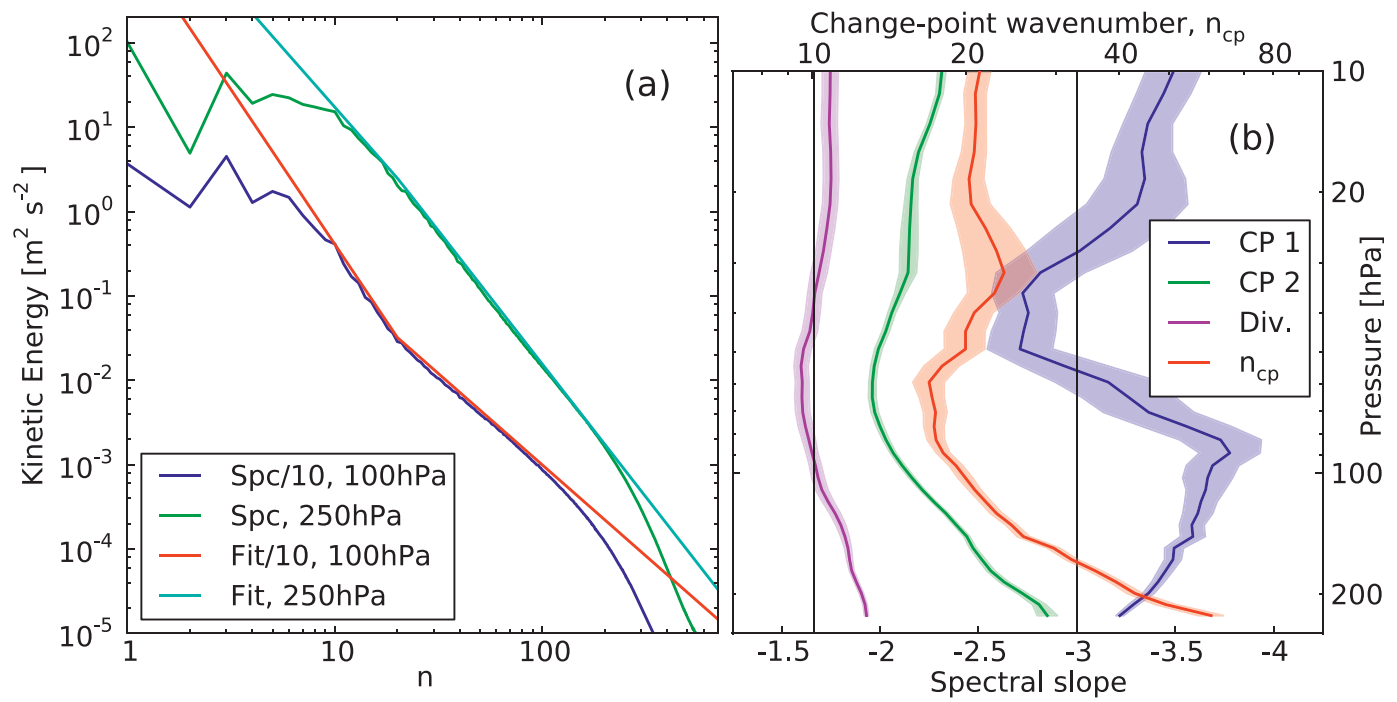

FIG. 2. (a) Spectra (Spc) and changepoint analyses (Fit) at 250 and $100 \mathrm{hPa}$. (The $100-\mathrm{hPa}$ spectrum is shifted down by 1 decade.) The changepoint fit is done over the range $n=10-100$. (b) The slope of the two changepoint segments (CP1 and CP 2), the divergent kinetic energy spectral slope (Div.), and the changepoint wavenumber $n_{\mathrm{cp}}$ as functions of altitude, with shading representing $95 \%$ confidence intervals. The top axis shows the changepoint wavenumber. The black vertical lines correspond to slopes of $-5 / 3$ and -3 .

kinetic energy over the range $250-100 \mathrm{hPa}$, which corresponds to the transition from troposphere to stratosphere over the global atmosphere.

Figure 2a illustrates the application of changepoint analysis to the spectra over the range $n=10-100$ at 250 and $100 \mathrm{hPa}$, showing the very clean scaling properties of these spectra (with excellent fits to power laws), and the sharpness of the spectral break at $100 \mathrm{hPa}$. This gives us confidence in the use of this diagnostic. The spectral break's location, which is calculated as part of the regression, determines the ranges over which the steep and shallow power laws extend. As evident in Fig. $2 \mathrm{~b}$, the break (red curve) moves upscale from $n \approx 60$ at $226 \mathrm{hPa}$ to $n \lesssim 20$ just above $100 \mathrm{hPa}$, before increasing slightly and remaining fairly stable around $n=$ $20 \mathrm{up}$ to $10 \mathrm{hPa}$. The slope of the steep, large-scale range (blue curve) varies around -3 with altitude. For this regime, the fit is done over a limited spectral range, resulting in large uncertainty and a wide $95 \%$ confidence interval (shaded region). The slope of the shallow, mesoscale range (green curve) is well defined (note the comparatively narrow confidence interval), and distinctly steeper than $-5 / 3$, which is the slope found by Nastrom and Gage (1985). The mesoscale slope decreases rapidly through the transition region to -2 around $60 \mathrm{hPa}$, before increasing slightly and remaining fairly stable up to $10 \mathrm{hPa}$. The divergent kinetic energy spectral slope (Div.), discussed later, is also shown.

The strong altitude dependence of the spectral break's location within the transition region $230-100 \mathrm{hPa}$, and of the shallow mesoscale slope, means that comparisons with other datasets in this altitude range must be performed with care. In particular, quantitative comparison with the Nastrom-Gage spectrum is problematic because the aircraft data included cover $250-150 \mathrm{hPa}$, which lies within our transition region, in which the ECMWF spectral break varies between $n \approx 30$ and $n \approx$ 60. Nastrom and Gage (1985) identified well-defined power laws for wavelengths larger than $1000 \mathrm{~km}(n \leq$ $40)$ and smaller than $400 \mathrm{~km}(n \geq 100)$, with a gradual transition in between (see their Fig. 3), which is in marked contrast to the sharp transition found here at particular vertical levels. Our results suggest the gradual transition found by Nastrom and Gage may result from averaging over vertical levels. The upper-tropospheric spectral break in the analysis occurs at larger scales than the break observed in aircraft data; we comment on this later.

The shallow spectral regime in the analysis at stratospheric altitudes is also consistent with kinetic energy spectra obtained by Bacmeister et al. (1996) from highaltitude research aircraft near $50 \mathrm{hPa}$, though again with slope steeper than the observed $-5 / 3$. Bacmeister et al. (1996) found no spectral break; this is consistent with the fact that at $50 \mathrm{hPa}$ the spectral break in the IPY analysis occurs at much larger scales $(n=20)$ than the largest scale $(n=60)$ resolved in the Bacmeister et al. spectra.

A direct comparison is possible with results from the high-resolution SKYHI general circulation model. Figure 1 of Koshyk and Hamilton (2001) shows a spectral break around $1000 \mathrm{~km}(n=40)$ at $211 \mathrm{hPa}$. (They 

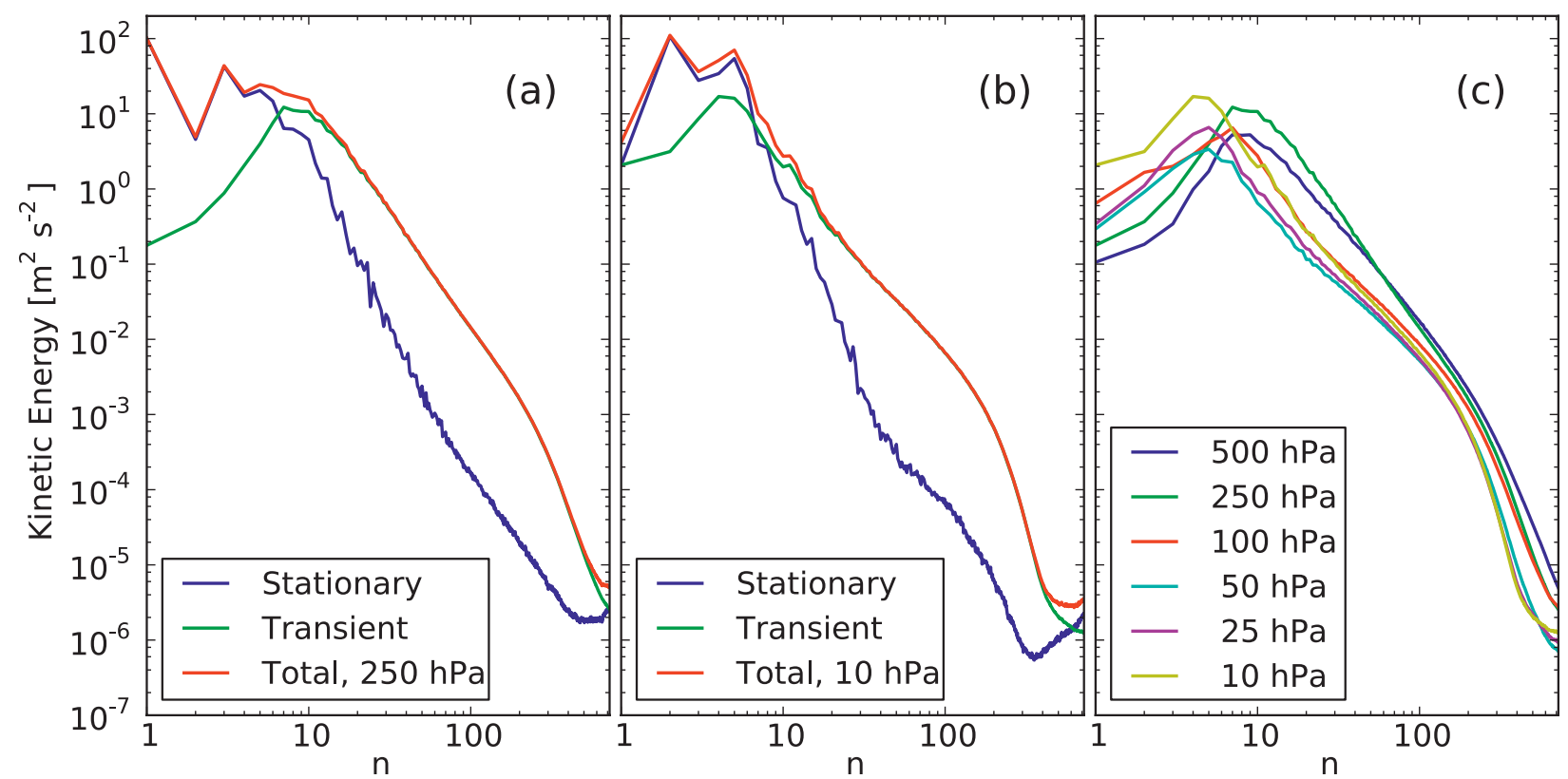

FIG. 3. Stationary and transient contributions to the kinetic energy spectrum at (a) 250 and (b) $10 \mathrm{hPa}$; the total spectrum has been added for comparison. (c) Transient kinetic energy at selected pressure levels.

state $500 \mathrm{~km}$, but $1000 \mathrm{~km}$ seems more consistent with their figure.) This is similar to what we find at $200 \mathrm{hPa}$, though of slightly larger scale. Figure 3 of Koshyk et al. (1999) shows a stratospheric average, but since this average is pressure weighted it is dominated by levels around $100 \mathrm{hPa}$. The distinct spectral break around $n=$ 20 found there is very consistent with our results at $100 \mathrm{hPa}$.

Hamilton et al. (2008) analyzed results from the Earth Simulator atmospheric GCM and found agreement between one-dimensional spectra at $200 \mathrm{hPa}$ and the Nastrom-Gage spectrum, with a smoother transition than found in either the IPY analysis or by Koshyk and Hamilton (2001). However, we note that they tuned their horizontal hyperdiffusion to obtain the observed spectrum, and that they had relatively coarse vertical resolution: 24 levels as opposed to 91 levels in the ECMWF IPY analysis.

Earlier deterministic versions of the ECMWF T799 forecast model did not exhibit mesoscale shallowing. Palmer (2001) and Palmer et al. (2005) in fact noted that the tropospheric kinetic energy spectrum produced by this model steepened in the mesoscale, rather than shallowing. With the addition of stochastic backscatter, a shallower mesoscale spectrum appeared (Palmer et al. 2005). Note, however, that stochastic backscatter is not used in the deterministic forecast model or ensemble data assimilation (N. Wedi 2012, personal communication), so the shallowing we observe here is due to other factors.
In IFS Cycle 32r3, with which the January 2008 IPY data studied here was generated, the ECMWF convective scheme was changed from a moisture-dependent to a relative-humidity-dependent organized entrainment rate (Hirons et al. 2012). This led to a better representation of the transition from shallow to deep convection, with increased cumulus congestus in the midtroposphere and decreased deep convection into the upper troposphere (Hirons et al. 2012). This may have led to a more energetic mesoscale spectrum with a stronger divergent component. Hamilton et al. (2008) also reported high sensitivity of the magnitude of divergent kinetic energy (but not spectral slope) to the cumulus scheme. In light of the mesoscale shallowing we find here, the current ECMWF analysis appears to be more realistic than earlier ECMWF analyses in terms of mesoscale variability.

Figure 3 shows a stationary-transient decomposition of the kinetic energy spectrum. In the upper troposphere (Fig. 3a), the transient component strongly dominates the stationary component for $n>10$, as found by Boer and Shepherd (1983). In the middle stratosphere (Fig. 3b), however, the transient component is less dominant around $n=10$ : the strong decrease in kinetic energy seen in Fig. 1 at these scales in the stratosphere is associated with the transient component of the flow. Figure $3 \mathrm{c}$ shows that the peak in the transient kinetic energy spectrum correspondingly moves from synoptic scales $(n=8)$ in the troposphere to planetary scales $(n=5)$ in the stratosphere. This is consistent with the mechanism of Charney-Drazin filtering (Charney and 


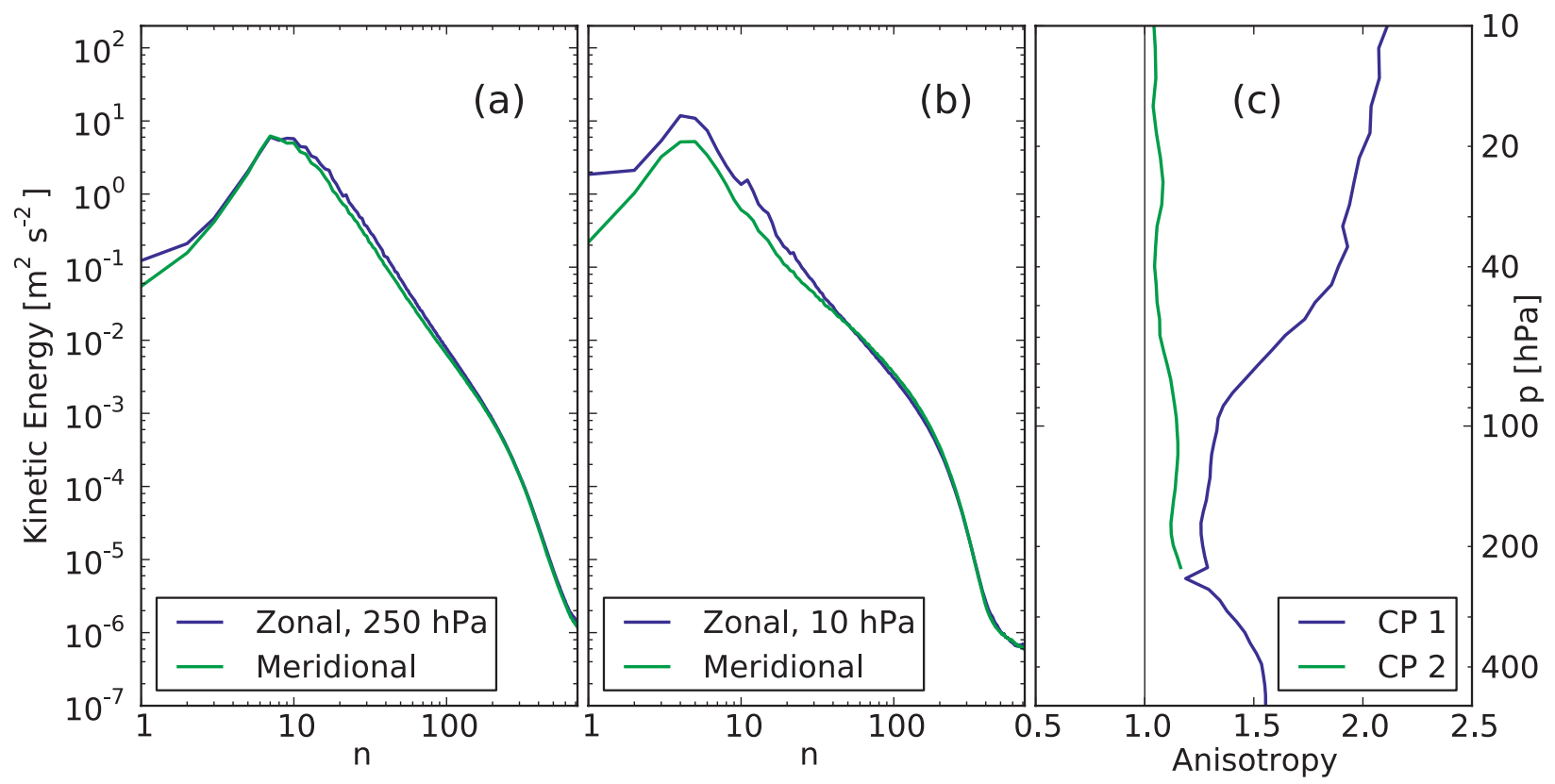

FIG. 4. Zonal and meridional components of the transient kinetic energy spectrum at (a) 250 and (b) 10 hPa. (c) Horizontal anisotropy, defined as the ratio of zonal to meridional transient kinetic energy, averaged over the first and second segments of the changepoint fit (CP 1 and CP 2).

Drazin 1961), which causes the amplitude of synopticscale evanescent waves to decay exponentially with altitude.

The transient kinetic energy is further decomposed into zonal and meridional components in Fig. 4. We exclude the stationary component, which is overwhelmingly zonal, from this decomposition. As noted by Boer and Shepherd (1983), transient flow in the upper troposphere (Fig. 4a) is almost isotropic, as expected for twodimensional turbulence, with approximate equipartition between zonal and meridional components, albeit slight zonal anisotropy. Boer and Shepherd (1983) decomposed the total kinetic energy into zonal and meridional components; however, since total kinetic energy approximately corresponds to transient kinetic energy for $n>8$, comparing our decomposition with theirs is valid. Shepherd (1987) showed that this approximate equipartition of transient kinetic energy applied at $n<8$ as well, as seen here. In the middle stratosphere (Fig. 4b), however, the large-scale regime becomes more zonally anisotropic, with a factor of two difference between zonal and meridional kinetic energy at the transient energy peak, while the mesoscale regime becomes more isotropic. To quantify this, Fig. 4c shows the ratio of zonal to meridional kinetic energy as a function of altitude for the two spectral ranges identified by changepoint analysis.

In Fig. 5, the kinetic energy is decomposed into rotational and divergent components. At $250 \mathrm{hPa}$ in the upper troposphere (Fig. 5a), the rotational dominates the divergent component by at least an order of magnitude, except within the dissipation range, and the two components scale very differently with $n$. In contrast, in the lower stratosphere $(100 \mathrm{hPa})$ the divergent component in the mesoscale $(n>40)$ is $2-3$ times larger than the rotational component, as shown in Fig. 5b: the two components are more comparable here than at large scales, and scale similarly in this range. This is like what was seen in the SKYHI general circulation model-see Fig. 4 of Koshyk et al. (1999). Balanced dynamics imply that divergent kinetic energy is much less than rotational kinetic energy - this holds for both the small Froude number balance possible in the mesoscale and the small Rossby number balance relevant to synoptic scales (Saujani and Shepherd 2006). Hence, we may interpret the regime in which the rotational component dominates the divergent component as being balanced, and the regime in which the divergent component is comparable to or larger than the rotational component as being unbalanced. (Note that unbalanced flow generally contains both divergent and rotational components, so a significant rotational contribution to the mesoscale spectrum is consistent with interpreting this flow as unbalanced.) With this interpretation, both upper troposphere and lower stratosphere exhibit a steep, balanced spectrum coexisting with a shallow, unbalanced spectrum. Given sufficient resolution, the two spectra must cross at some scale, leading to a spectral break. However, 


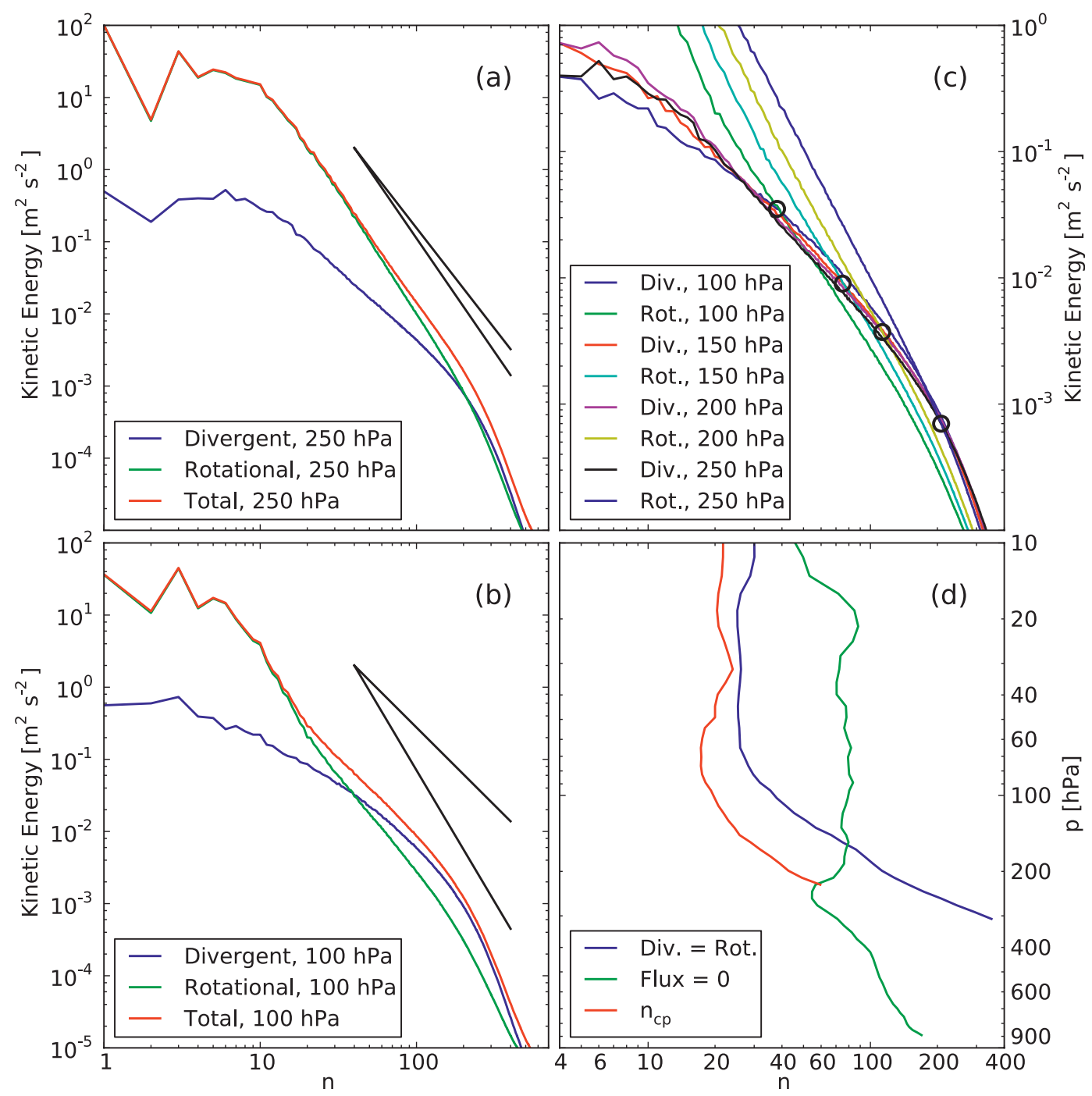

FIG. 5. Divergent, rotational, and total components of the kinetic energy spectrum at (a) $250 \mathrm{hPa}$, with lines of slope -2.79 and -3.15 for comparison, and (b) $100 \mathrm{hPa}$, with lines of slope -2.16 and -3.65 for comparison. (c) A blowup of the transition region, with the intersections of the rotational and divergent spectra on given levels circled in black. (d) The wavenumber at which the divergent and rotational components intersect as a function of height; the wavenumber at which the kinetic energy flux reverses sign and the changepoint wavenumber also appear.

in light of the finite resolution imposed by the dissipation range, the unbalanced spectrum is sufficiently weak at $250 \mathrm{hPa}$ that it fails to emerge in the total energy, while at $100 \mathrm{hPa}$ it is sufficiently strong, relative to the balanced spectrum, that it emerges in the total energy for $n>20$.

Figure $5 \mathrm{c}$ shows a blowup of the transition region $230-100 \mathrm{hPa}$, with the intersections of the rotational and divergent spectra indicated by open circles. The intersections move rapidly to larger scale as altitude increases; this results from decreased rotational power rather than increased divergent power, and is consistent with the mechanism of Charney-Drazin filtering referred to in connection with Fig. 3. As evident in Fig. 5d, the vertical dependence of the wavenumber where the divergent and rotational spectra intersect closely parallels that of the spectral break in the total kinetic energy spectrum. The mesoscale spectrum shallows predominantly because the shallower divergent spectrum makes a relatively large contribution to the total kinetic energy at mesoscales. Hence, our interpretation is that the spectral break reflects a transition from a balanced spectral range at large scales to an unbalanced spectral range in the mesoscale, and the break's upscale shift over the transition region $230-100 \mathrm{hPa}$ results from preferential decrease of synoptic-scale balanced kinetic 

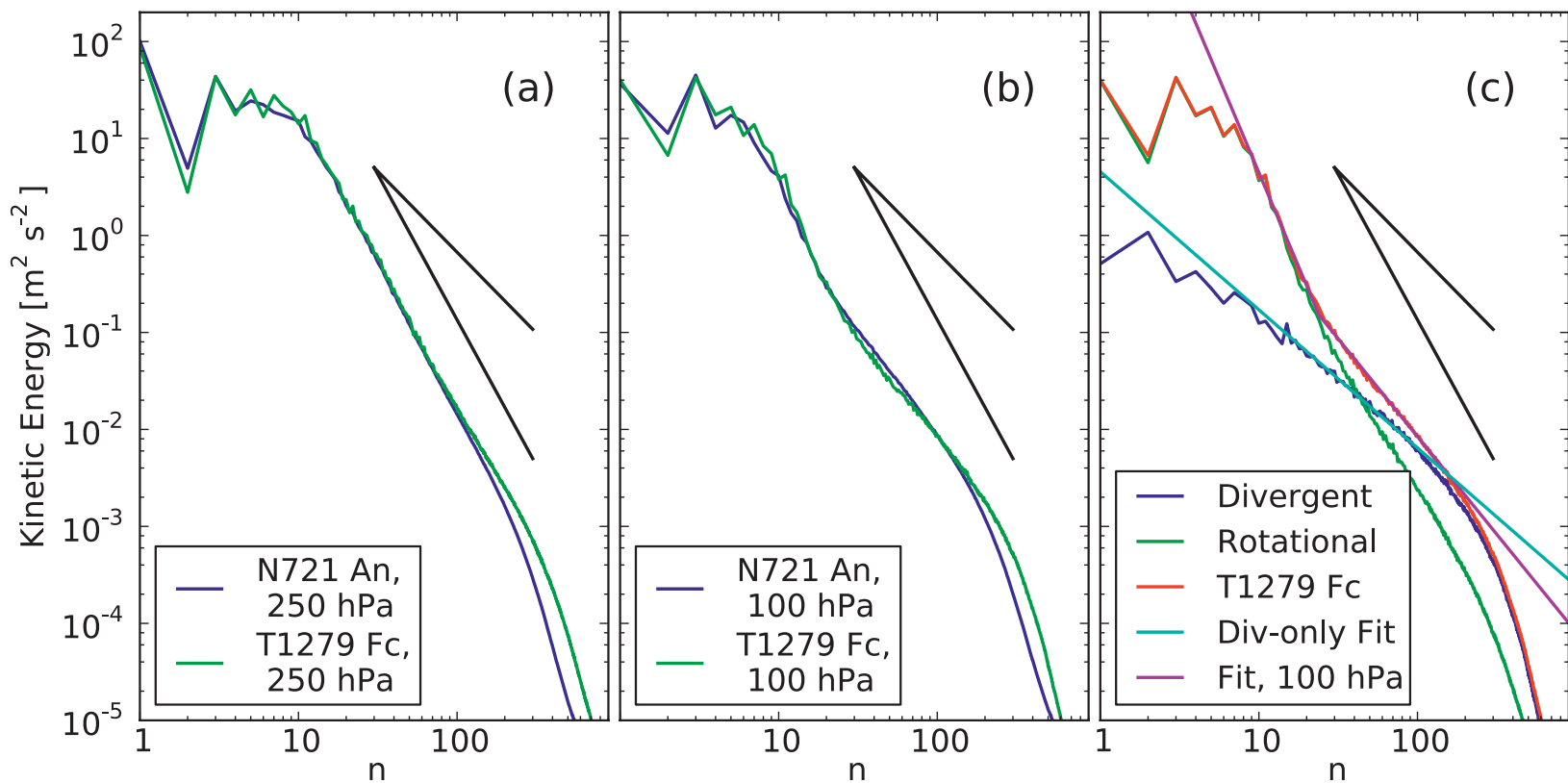

FIG. 6. Kinetic energy spectra at (a) 250 and (b) $100 \mathrm{hPa}$ for T1279 forecast (Fc) and the IPY analysis (An) data. The lines have slopes of -3 and $-5 / 3$. (c) The T1279 forecast spectrum at $100 \mathrm{hPa}$ decomposed into rotational and divergent components, with a changepoint fit (Fit) and a fit to the divergent spectrum (Div-only Fit). The slope of the divergent spectrum is -1.4 , shallower than the $-5 / 3$ found in the IPY analysis.

energy with height. Our unbalanced mesoscale is consistent with Bartello (2010), who simulated idealized rotating and stratified turbulence and found that a shallow mesoscale range emerged as a consequence of ageostrophic modes, which included all divergent effects, exceeding the geostrophic (balanced) component of the kinetic energy spectrum.

As noted earlier, the spectral break in the analysis occurs at larger scales than found by Nastrom and Gage (1985). Because the steep balanced part of the spectrum is presumably well constrained by observations, this suggests excess power in the unbalanced flow, and that the shallow mesoscale spectrum is overly energetic in the analysis, at least in the vicinity of the transition wavenumber. Excess energy in the unbalanced flow would cause the divergent to intersect the rotational spectrum at larger scales, and the spectral break to appear at larger scales. On the other hand, the difference could reflect sampling issues, because our spectra likely include more contributions from the tropics, where the unbalanced component of the flow is expected to be stronger, than do the Nastrom-Gage spectra.

As shown in Fig. 2b, linear fits to the divergent kinetic energy spectrum over the range $n=20-100$ above $100 \mathrm{hPa}$ reveal a slope (Div.) very close to $-5 / 3$, which is the slope observed in aircraft data. This suggests the mesoscale spectrum and shallowing are not properly resolved in the analysis; up to $n=100$, the steeper rotational spectrum still significantly contributes to the slope, leading to a steeper spectrum, and beyond $n=$ 100 , the divergent spectrum itself begins to steepen, presumably because of dissipation. If the $-5 / 3$ power-law behavior of the divergent spectrum extended to larger $n$ before encountering dissipation, as presumably would happen in higher-resolution data, while simultaneously the rotational kinetic energy continued to drop off, the divergent spectrum and its $-5 / 3$ power law would dominate at some $n$. In such a case, the changepoint analysis would recover a mesoscale spectrum with slope closer to $-5 / 3$.

To test this hypothesis, we examine kinetic energy spectra from forecasts produced with a more recent, higher-resolution (T1279) version of the ECMWF forecast system (Fig. 6). The results are nearly the samemesoscale shallowing again emerges as a result of the divergent exceeding the rotational component of the flow, as seen in Fig. 6c-but the mesoscale spectrum is slightly weaker, has a somewhat shallower slope ( -2 in the T1279 forecast as opposed to -2.16 in the IPY analysis at $100 \mathrm{hPa}$ ), and extends to higher wavenumbers. (The dissipation range appears to begin somewhere between $n=200$ and $n=300$.) All of these features are more realistic, as might be expected from a higher-resolution analysis. The divergent kinetic energy spectrum also has slope -1.4 , which is shallower than the $-5 / 3$ found in the IPY analysis. This suggests 


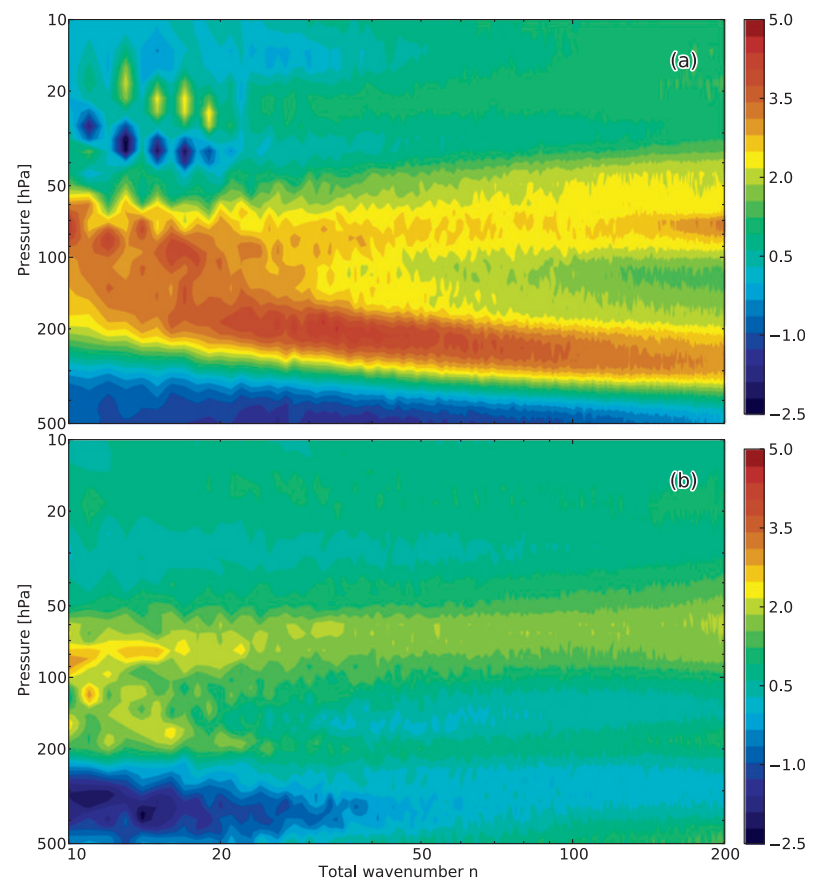

FIG. 7. The relative vertical derivative (i.e., the derivative scaled by the local value) of density-weighted (a) rotational and (b) divergent kinetic energy. This is a measure of relative attenuation with height. The color bar corresponds to the value.

that the appearance of a changepoint transition to a shallower mesoscale slope in the IPY analysis is a generic feature of the latest ECMWF forecast models, but the exact details, such as the wavenumber of the transition and the slope of the mesoscale spectrum, can be expected to depend on the forecast model.

The relative decrease of rotational and divergent energy is shown in Fig. 7 as a function of pressure and wavenumber. By relative decrease, we mean the vertical derivative of density-weighted kinetic energy scaled by the local value,

$$
\text { relative decrease }=-\frac{\partial \log \left(\rho E_{n}\right)}{\partial \log p},
$$

where $\rho$ is density and $p$ pressure. This measures relative attenuation with height. Figure 7 shows relative decrease except for rotational kinetic energy at the largest scales in the stratosphere, especially in the band $50-20 \mathrm{hPa}$. The increase of rotational kinetic energy at these scales and pressure levels is consistent with Fig. 1. Rotational kinetic energy exhibits a significant decrease above $250 \mathrm{hPa}$ at the larger scales, as already discussed. Above $100 \mathrm{hPa}$, the relative decreases are similar between the rotational and divergent kinetic energy. Since unbalanced flow contains both rotational and divergent components, this suggests these decreases are associated with the same (i.e., unbalanced) component of the flow. The relative decreases are also largely scale independent, which explains why the shallow mesoscale power-law scaling can be maintained with approximately the same exponent above $100 \mathrm{hPa}-$ see Fig. 2b. Why the relative decreases should be scale independent is unclear.

\section{Nonlinear interactions and spectral fluxes}

Figure 8 shows the rotational kinetic energy nonlinear interaction terms for various levels. A negative value at a given scale indicates loss of energy to other scales, while a positive value indicates gain from other scales. The integral over all wavenumbers is zero by construction. In the upper troposphere ( 350 and $250 \mathrm{hPa}$ ), there is net loss of energy from $n>10$ and net gain at two distinct scales, $n=3$ and $n=8-9$, as shown in Fig. 8a, implying upscale transfer of rotational kinetic energy to those scales. Interaction terms represent exchanges with all other scales. However, since, for example, at $250 \mathrm{hPa}$ the terms only reach large negative and positive values at synoptic and planetary scales, respectively, energy exchanges must be predominantly between those scales. Both immediately above and below these pressure levels, the nonlinear transfers are much weaker. The double-peaked structure at the largest scales differs from the single peak at $n=3$ found by Boer and Shepherd (1983). Of course, unlike spectra, nonlinear interaction terms even at the largest scales include contributions from spectrally nonlocal wavenumber triads involving smaller scales, and are sensitive to spatial resolution. This is illustrated by the fact that Boer and Shepherd (1983) found positive interaction terms at the smallest resolved scales (around $n=30$ ), which is almost certainly an artifact of missing nonlinear interactions with slightly smaller scales [see discussion in Boer and Shepherd (1983)]. With these much-higherresolution analyses, the interaction terms for $n>10$ remain negative to $n=60$. We will discuss convergence further when we examine nonlinear fluxes at the higher wavenumbers.

In the stratosphere (100 hPa and above), the synopticscale kinetic energy source region $n>10$ disappears, which is consistent with the rapid decrease of synopticscale kinetic energy between 250 and $100 \mathrm{hPa}$ seen in the kinetic energy spectra (section 3). The interaction terms now show upscale transfer within the planetaryscale range $n<10$, which grows substantially with altitude, becoming especially pronounced at 10 and $5 \mathrm{hPa}$. We comment further on this when we discuss zonal mean-eddy decompositions of the spectral transfers. 

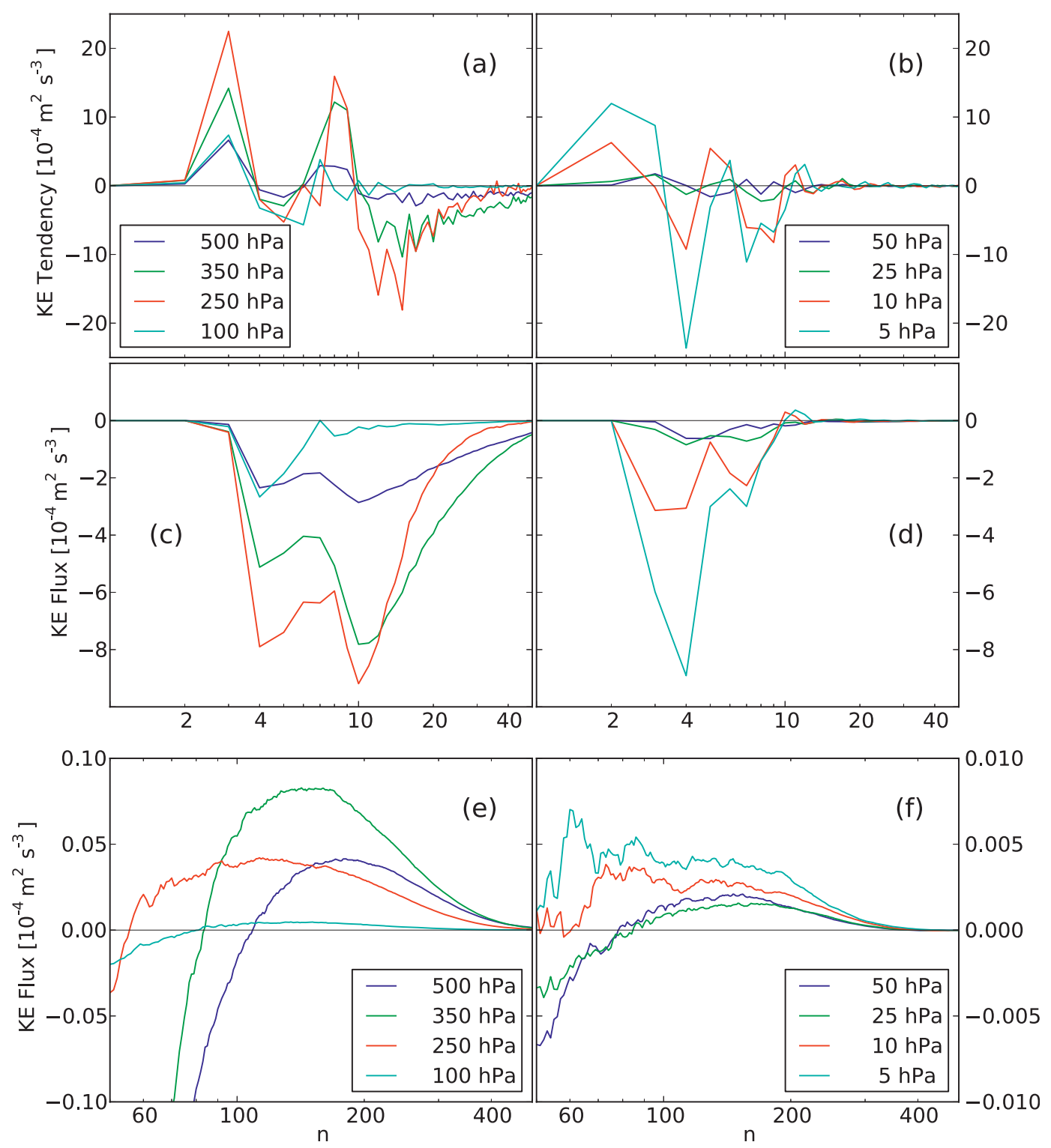

FIG. 8. Rotational nonlinear interaction terms and kinetic energy fluxes: (a),(b) nonlinear transfers between planetary and synoptic scales, (c),(d) fluxes at planetary and synoptic scales, and (e),(f) fluxes at the synoptic scale and mesoscale. Note the different scalings on the vertical axes, and that the range of the horizontal axis for (e) and (f) differs from that for (a)-(d). The plots in (a),(c), and (e) show transfers and fluxes in the tropopause and uppertroposphere-lower stratosphere region, while (b),(d), and (f) show them in the stratosphere. The interaction terms have been scaled by wavenumber to preserve the integral.

The remaining panels of Fig. 8 show nonlinear fluxes computed from (11) and (12). These represent spectral transfer of rotational kinetic energy (upscale for negative fluxes, downscale for positive fluxes) past a given wavenumber due to nonlinear interactions. Starting from the barotropic vorticity equation, one cannot define for the total kinetic energy a spectral flux that is a sum over compensating interactions and goes to zero at the truncation scale. The approach taken here is to restrict consideration to the rotational component of the flow. This allows us to define spectral fluxes and is complementary to the enstrophy flux analysis, which of course solely involves the rotational flow. This does not provide a complete representation of the energy budget, but other terms are missing in any case (e.g., baroclinic conversions), and this framework does allow one to investigate the nonlinear spectral fluxes relevant to two-dimensional turbulence. In particular, 


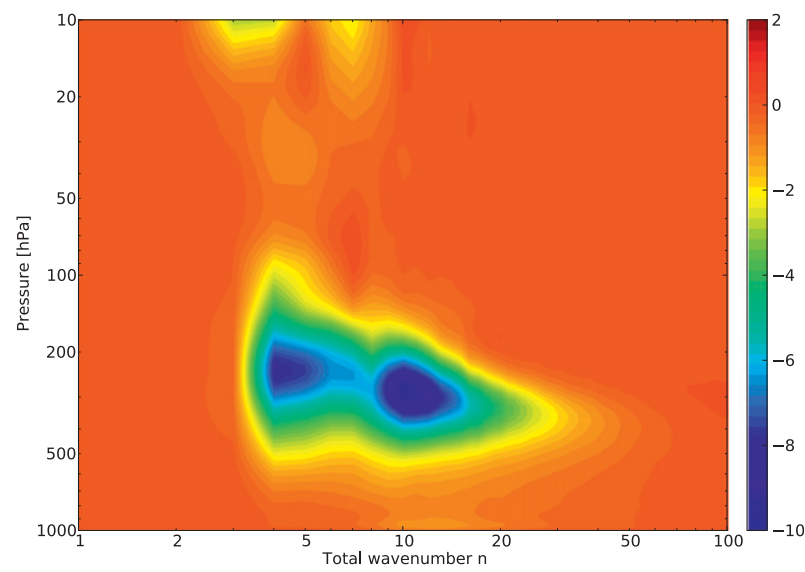

FIG. 9. Rotational kinetic energy fluxes $\left(\mathrm{m}^{2} \mathrm{~s}^{-3}\right)$ scaled by a factor of $10^{4}$.

it allows us to reexamine with a higher-resolution analysis earlier results on rotational fluxes, which is in itself of interest given the sensitivity of nonlinear interactions and fluxes to small-scale structure and spectral truncation.

The large-scale portions of these plots-Figs. $8 \mathrm{c}$ and dshow what we could already conclude from the nonlinear interaction terms: an upscale kinetic energy flux, maximizing in the upper troposphere and again in the upper stratosphere. The downscale energy fluxes at the smaller scales-Figs. 8e and f-are at least two orders of magnitude smaller than the upscale fluxes, whereas in Boer and Shepherd (1983) they were only a factor of three smaller. This confirms their hypothesis that the strong inferred downscale energy fluxes were artifacts of limited spectral resolution. It also shows that even the T106 analysis of Straus and Ditlevsen (1999), for which the (vertically integrated) downscale energy flux was about $10 \%$ of the upscale flux, did not fully resolve the upscale energy transfer. The higher-resolution analysis used here confirms that kinetic energy transfer is overwhelmingly to larger scale. Nevertheless, the downscale energy fluxes appear to be resolved in the sense that there are spectral ranges of nearly constant positive flux, at least in the upper troposphere and stratosphere ( $250 \mathrm{hPa}$ and above), suggesting that the kinetic energy sources are spectrally confined and captured by the analysis. In particular, the kinetic energy flux is downscale in the shallow mesoscale regime. At lower altitudes, the flux maximum appears to be limited by the dissipation range evident in the spectra for $n>100$.

Figure 9 shows the kinetic energy flux's vertical structure, highlighting the concentration of upscale flux around the tropopause. Figure 11 of Boer and Shepherd
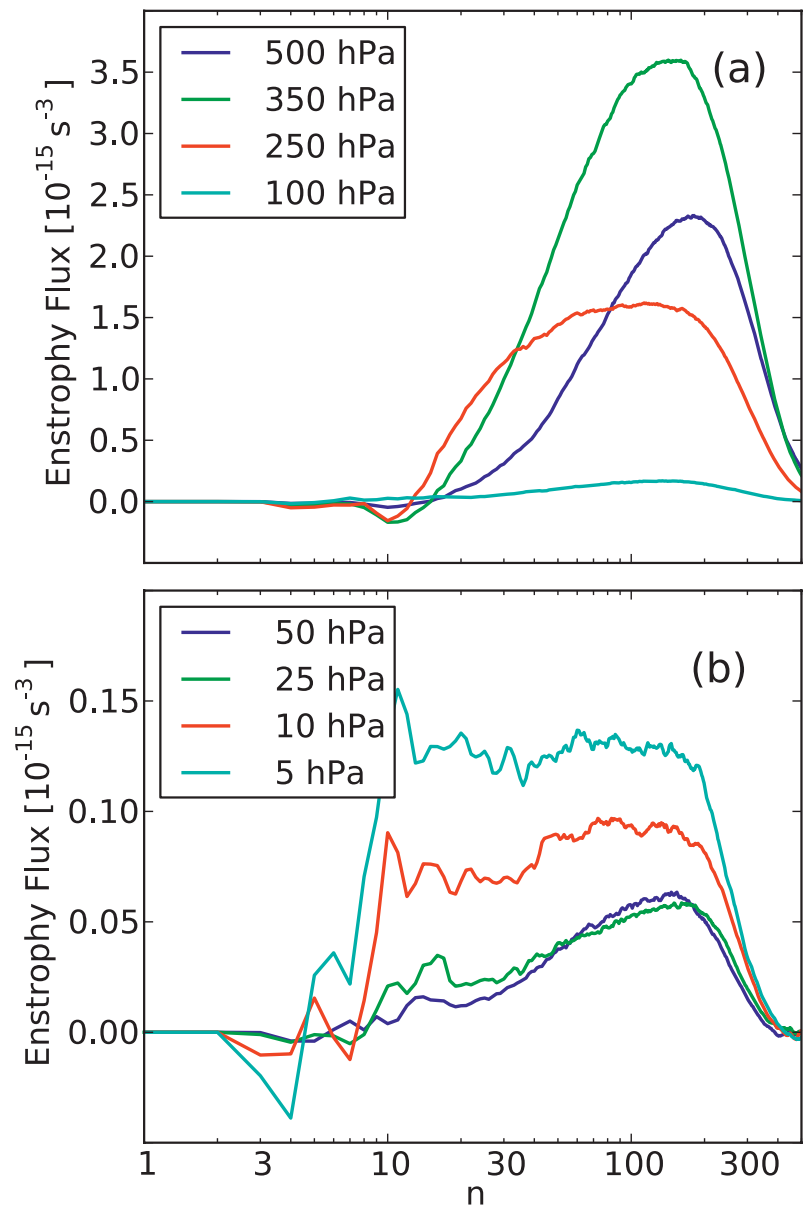

FIG. 10. Enstrophy flux at selected pressure levels. Note the different scales on the vertical axes.

(1983) is similar, but here the flux is almost entirely upscale for the range shown, and has two peaks.

Nonlinear interactions of the rotational flow conserve both kinetic energy and enstrophy, so spectral fluxes of enstrophy can also be defined, and are shown in Fig. 10. These are overwhelmingly downscale, as found by Boer and Shepherd (1983) and Straus and Ditlevsen (1999). In the middle troposphere (500 and $350 \mathrm{hPa})$, the enstrophy fluxes increase with wavenumber until turning over sharply, indicating energy/enstrophy generation over a wide spectral range, limited only by the dissipation range of the analysis. At these altitudes, the maximum enstrophy flux presumably has not converged. In the upper troposphere $(250 \mathrm{hPa})$, in contrast, the enstrophy flux is approximately constant for $n=50-200$, which is consistent with expectations for two-dimensional turbulence and a spectrally localized source. The kinetic energy slope is also approximately -3 at these altitudes (see Fig. 1), the slope expected for a twodimensional enstrophy-cascading turbulent subrange. 


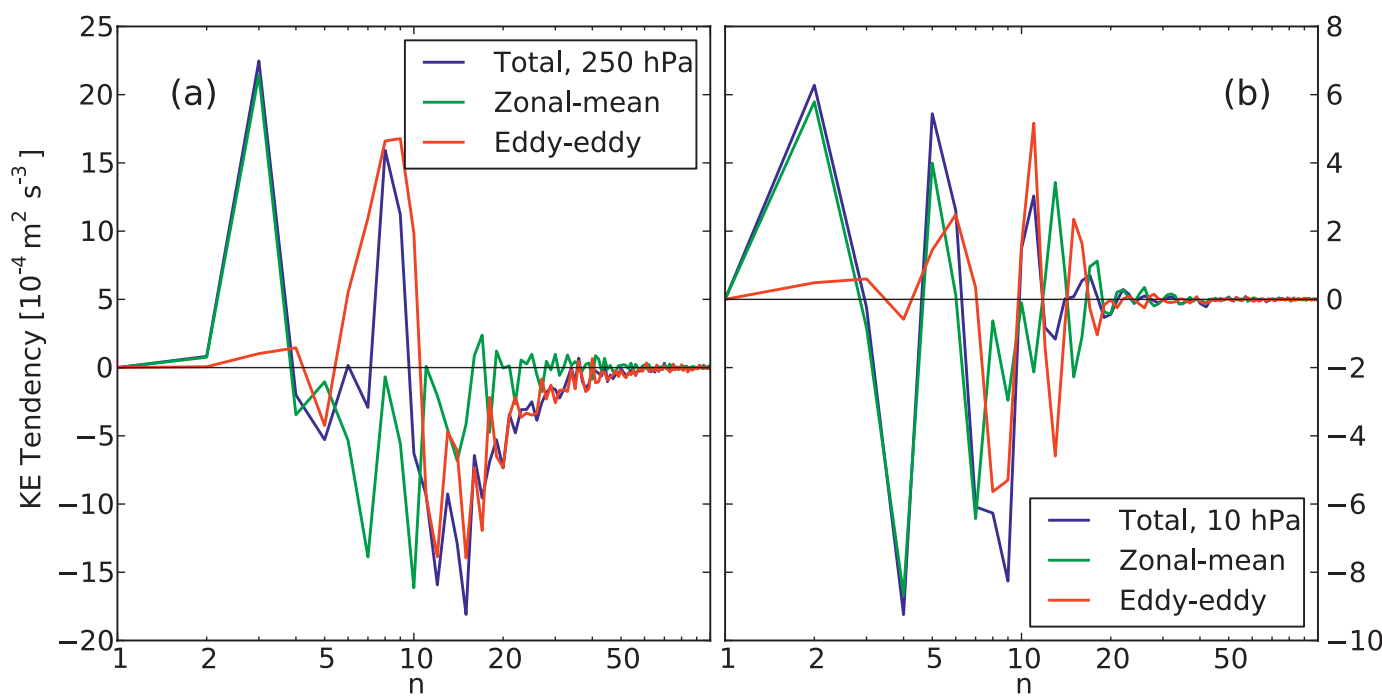

FIG. 11. Total, zonal mean-eddy, and eddy-eddy nonlinear rotational kinetic energy interaction terms at (a) 250 and (b) $10 \mathrm{hPa}$. The interaction terms have been scaled by the wavenumber to preserve the integral.

This is also the altitude where the kinetic energy flux changes sign (to positive) at the largest scale, as shown in Fig. 5d. Downscale contributions to the kinetic energy flux begin to dominate upscale contributions at the wavenumber where the flux changes sign. (Note that downscale and upscale fluxes will generally coexist in the same spectral range.) A sign change at large scales is consistent with a spectrally confined injection region for the downscale flux and with constant enstrophy flux.

Notably, Cho and Lindborg (2001) estimated a downscale enstrophy flux of $2 \times 10^{-15} \mathrm{~s}^{-3}$ from structurefunction analysis of aircraft data in the stratospheric polar vortex at altitudes of 9-12 km $(\sim 200 \mathrm{hPa})$. While we cannot compare directly with our global estimates, the value is similar to our calculated enstrophy flux of $1.5 \times 10^{-15} \mathrm{~s}^{-3}$ at $250 \mathrm{hPa}$.

The lower stratosphere (50 and $25 \mathrm{hPa}$ ) resembles the middle troposphere in that the enstrophy flux increases steadily with wavenumber until limited by the dissipation range, again implying energy/enstrophy generation over a wide range of scales. In the upper stratosphere (10 and $5 \mathrm{hPa}$ ), however, a well-developed downscale enstrophy cascade emerges, consistent with a spectrally localized planetary-wave enstrophy source, and complementing strong upscale energy fluxes at these altitudes.

Shepherd (1987) emphasized the mean flow's important role in inducing tropospheric spectral transfers, suggesting that zonal mean-eddy interactions accomplished roughly half the upscale energy flux, and eddyeddy interactions (possibly described by homogeneous two-dimensional turbulence theory) the other half. To better understand our diagnosed transfers, we therefore decompose them into zonal mean and eddy contributions.
This is essentially equivalent to Shepherd's stationarytransient decomposition, since the stationary flow is mostly zonal. We focus on 250 and $10 \mathrm{hPa}$, which exhibit strong upscale energy fluxes.

Figure 11 shows the energy interaction terms. The two peaks found earlier at $250 \mathrm{hPa}$ have distinct origins: the peak around $n=8$ is sourced by eddy-eddy interactions and draws from $n=10-20$, while the peak around $n=3$ is sourced by zonal mean-eddy interactions and draws mainly from scales around $n=8$. The resulting upscale energy flux, shown in Fig. 12a, thus exhibits a "hand off," with eddy-eddy interactions carrying energy up to $n=8$, and zonal mean-eddy interactions carrying it the rest of the way up to $n=3$. The same feature was identified in much-lower-resolution analyses by Shepherd (1987). In contrast to Shepherd (1987), however, here the downscale enstrophy flux is overwhelmingly carried by eddy-eddy interactions, as seen in Fig. 12b. Hence the upscale energy flux in the upper troposphere reflects two distinct processes: eddy-eddy transfers, which can be understood, along with the downscale enstrophy flux, within the framework of twodimensional turbulence, and upscale zonal mean-eddy energy transfers, which are wave-mean flow interaction rather than homogeneous two-dimensional turbulence. The energy spectral slope in this range is not that expected for a two-dimensional turbulent inverse cascade; this can be attributed to the substantial contribution from zonal mean-eddy transfers to the upscale flux, as well as the limited spectral range over which the upscale energy flux develops.

At $10 \mathrm{hPa}$, the role of eddy-eddy interactions is diminished and that of zonal mean-eddy interactions 

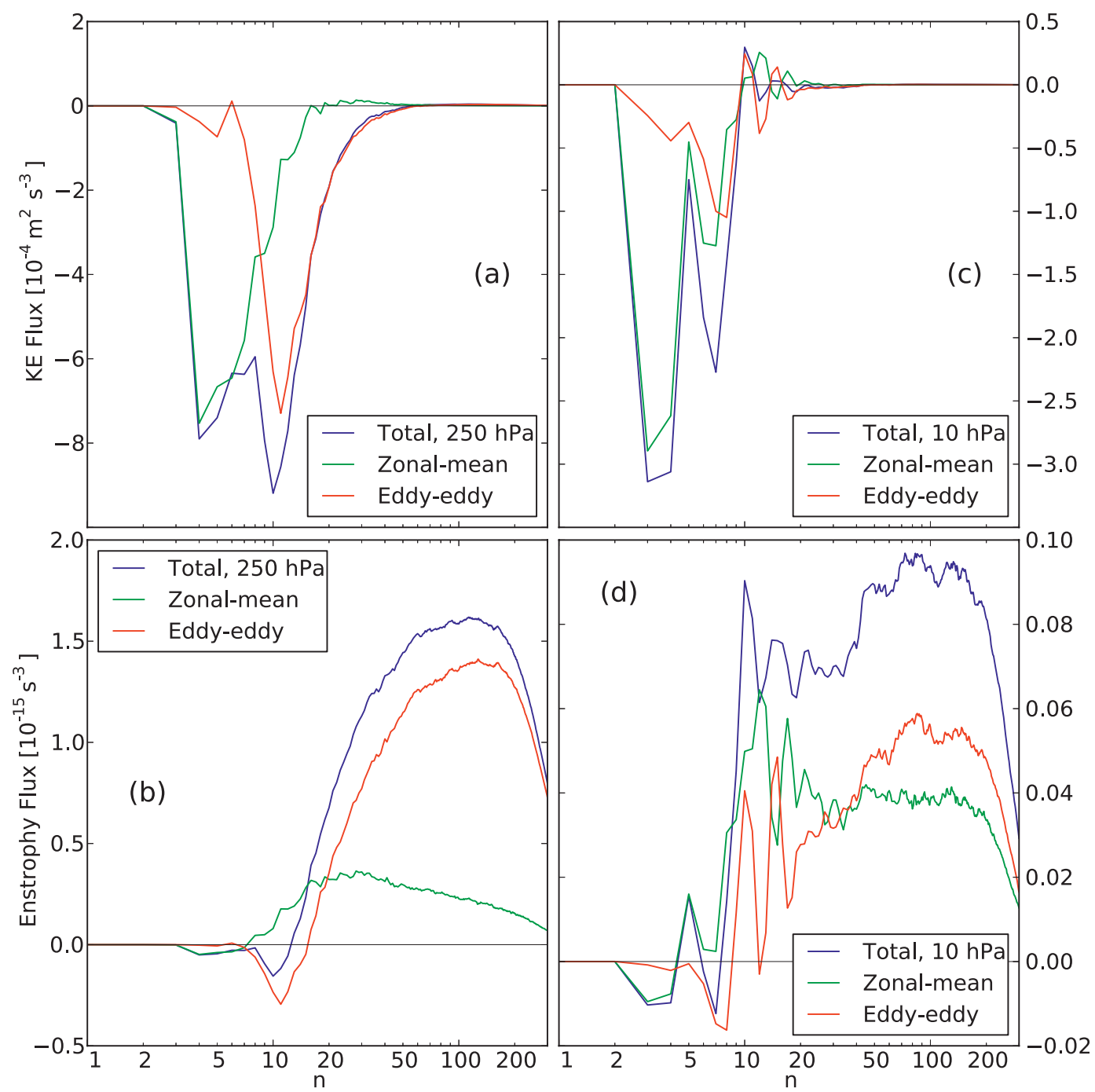

FIG. 12. (a),(c) Total, zonal mean-eddy, and eddy-eddy rotational KE fluxes, and (b),(d) enstrophy fluxes. The plots in (a) and (b) show $250 \mathrm{hPa}$, while (c) and (d) show $10 \mathrm{hPa}$.

enhanced, as shown in Fig. 12d. This is because at $10 \mathrm{hPa}$ the transient flow is much weaker than the overwhelmingly zonal stationary flow (Fig. 3). At these altitudes, the dynamics is most accurately represented as wave-mean flow interaction. Zonal mean-eddy interactions primarily drive the upscale energy flux, displayed in Fig. 12c, while even the downscale enstrophy flux has a sizeable contribution-roughly one-half-from the zonal mean-eddy interactions, as can be seen in Fig. $12 \mathrm{~d}$. Notably, this component of the flux is relatively constant for $n>20$. We thus see a downscale enstrophy cascade from a spectrally localized planetary-scale source, carried primarily by zonal mean-eddy interactions. The contribution of eddy-eddy interactions to the downscale enstrophy flux is far from negligible, and grows with $n$, highlighting the role of nonlinearity in wave breaking that drives the downscale enstrophy cascade in the stratospheric surf zone.

The behavior of the interaction terms and fluxes is qualitatively similar at 15 and $5 \mathrm{hPa}$, except that the zonal mean-eddy interactions strengthen with altitude. This is consistent with the fact that, as altitude increases, the polar jet strengthens, planetary wave amplitudes increase, and the surf zone gets more active.

\section{Summary and discussion}

The extent to which two-dimensional turbulence theory describes atmospheric circulation has been much investigated. Limited spatial resolution constrained earlier studies (Boer and Shepherd 1983; Trenberth and Solomon 1993; Straus and Ditlevsen 1999) of global 
atmospheric analyses. Recent state-of-the-art meteorological analyses have much higher spatial resolution and include well-resolved stratospheres, so reexamining this issue is of interest. We do so using the ECMWF "International Polar Year" T799 operational analysis.

Aspects of the meteorological analysis not well constrained by measurements will be largely determined by the forecast model. This consideration applies particularly to the mesoscale features of the analysis, and a relevant question then is whether the meteorological analysis appears realistic in light of direct observations and results from general circulation models. A case in point is the marked shallowing of the kinetic energy spectrum observed at mesoscales in upper-tropospheric aircraft observations (Nastrom and Gage 1985) and in general circulation models (Koshyk et al. 1999; Koshyk and Hamilton 2001). The ability to reproduce such a feature is a test of whether a meteorological analysis-and, by extension, its underlying forecast model — has a physically realistic level of mesoscale variability.

We first examine horizontal wavenumber spectra of total (rotational plus divergent) horizontal kinetic energy, from 500 to $10 \mathrm{hPa}$. The spectra fall off rapidly beyond $n \sim 100(\lambda \sim 400 \mathrm{~km})$, suggesting that this is the effective resolution of the analysis in this altitude range. In accord with earlier studies, we find a distinct power-law scaling, shown in Fig. 1a, at synoptic scales $(n>10)$, the slope varying from -2.7 to -3.7 with altitude, as seen in Fig. 2b. At $250 \mathrm{hPa}$, the slope is close to -3 , in agreement with Boer and Shepherd (1983) and Trenberth and Solomon (1993). In contrast to earlier studies, a distinct spectral break emerges above $250 \mathrm{hPa}$ - see Fig. 1b-with a shallower mesoscale spectrum as seen in upper-tropospheric aircraft data (Nastrom and Gage 1985). This shallow mesoscale spectrum was not observed in earlier deterministic versions of the ECMWF forecast model (Palmer 2001; Palmer et al. 2005). The mesoscale spectrum has a well-defined power law, generally between -2.0 and -2.5 , as displayed in Fig. 2a, which is steeper than the observed $-5 / 3$.

Changepoint analysis identifies the spectral break, which moves upscale from about $n=60(\lambda=650 \mathrm{~km})$ to $n=20(\lambda=2000 \mathrm{~km})$ between 230 and $100 \mathrm{hPa}$, as shown in Figs. $1 b$ and $2 b$, remaining fairly stable at higher altitudes. We expect this transition layer, and the range of wavenumbers at which the spectral break occurs, to be model dependent; in particular, for a higherresolution analysis with a shallower mesoscale spectrum, the break might appear at lower altitudes and higher $n$ than found here. Comparison with observations must be performed with care, since the location of the spectral break changes substantially with height. In particular, a quantitative comparison with the celebrated
Nastrom-Gage spectrum is not possible, since the aircraft data included in that analysis were obtained from flights over the $250-150-\mathrm{hPa}$ range. Averaging between pressure levels may account for the lack of such a sharp spectral break in their results. Over this altitude range, the spectral break in the ECMWF analysis varies from $n=60$ to $n=30$, which is broadly consistent with the transition region $n=40-100$ evident in the NastromGage spectrum. In the lower stratosphere $(50 \mathrm{hPa})$, the shallow mesoscale spectrum is consistent with the research aircraft observations of Bacmeister et al. (1996), though again with a somewhat steeper spectral slope than the $-5 / 3$ found in the observations.

Comparing the ECMWF IPY mesoscale spectrum with that of a higher-resolution ECMWF forecast model (Fig. 6) reveals that the mesoscale spectrum in the latter is slightly weaker and shallower, with a slope closer to $-5 / 3$ ( -2 in the T1279 data as opposed to -2.16 in the IPY analysis at $100 \mathrm{hPa}$ ). This suggests the shallow mesoscale spectrum in the IPY analysis is generic, but details such as power and slope are model dependent.

In accord with earlier studies, the transient component dominates the kinetic energy spectra (Fig. 3) except at the largest scales $(n<8)$. In the lower stratosphere, Charney-Drazin filtering removes the transient synopticscale energy, the transient component becomes less dominant, and the transient kinetic energy peak shifts to larger scales. While the stationary component of the flow is largely zonal, the transient component is almost isotropic between zonal and meridional components, as shown in Fig. 4, albeit with clear and more or less scale-independent zonal anisotropy, which applies to both the steep and the shallow spectral ranges. Largescale zonal anisotropy increases in the middle stratosphere, with twice as much zonal as meridional kinetic energy at the transient energy peak, while the mesoscale regime becomes more isotropic. Stronger zonal anisotropy in the large-scale transient flow reflects the stronger role of the mean flow, which makes the eddies more zonally anisotropic through shearing. The more isotropic mesoscale spectrum implies that zonal mean-eddy interaction is not important in this spectral range, dominated presumably by the gravity wave field.

Decomposing the spectra into rotational and divergent components (Fig. 5) shows that the spectral break seen in the total spectra distinguishes a "balanced" synoptic-scale regime, where divergent kinetic energy is much less than rotational kinetic energy, and an "unbalanced" mesoscale regime, where the two components are roughly comparable, consistent with general circulation model results (Koshyk et al. 1999; Koshyk and Hamilton 2001). In fact, divergent kinetic energy is larger than rotational kinetic energy in the 
mesoscale regime, but only by a factor of $2-3$, whereas at large scales rotational flow contains an order of magnitude more power than divergent flow. The spectral break's rapid upscale transition between 250 and $100 \mathrm{hPa}$ is due to preferential decay of synoptic-scale rotational kinetic energy (hence balanced flow) over this altitude range (Figs. 5 and 7). Above these levels, the energy decrease with altitude is roughly scale independent, and similar for both rotational and divergent flow, except for the largest rotational scales.

Linear fits to the divergent kinetic energy spectrum over the range $n=20-100$, displayed in Fig. 2b, show a slope above $100 \mathrm{hPa}$ close to the $-5 / 3$ slope observed in aircraft data. This suggests the IPY analysis does not properly resolve the mesoscale shallowing. Indeed, in the higher-resolution T1279 analysis, the divergent spectrum extends to larger $n$ before encountering the dissipation range, giving a slope closer to the observed value.

Our study next examines nonlinear interactions and spectral fluxes. We restrict consideration to the rotational component of the flow so we can define spectral fluxes that tend to zero in the truncation limit, and reexamine earlier results using a higher-resolution analysis. The potential importance of nonlocal wavenumber triads means that small-scale structure and spectral truncation might affect large-scale spectral transfers, so this is of interest.

The kinetic energy interactions show upscale energy flux to large scales (Fig. 8) consistent with earlier studies; however, the IPY analysis appears to resolve the source region well at $250 \mathrm{hPa}$ and above, in the sense that interactions become very small for $n$ well below the dissipation range, and the downscale energy flux at large $n$ is negligible (approximately two orders of magnitude smaller than the upscale flux). This confirms that significant positive rotational kinetic energy fluxes found in earlier studies, especially Boer and Shepherd (1983), were an artifact of limited resolution. Nonlinear fluxes maximize between 300 and $200 \mathrm{hPa}$ (Fig. 9)-large-scale turbulence centers on the extratropical tropopause. At these levels, the downscale enstrophy flux develops a pronounced plateau, indicating an enstrophy-cascading inertial subrange (Fig. 10). In previous studies, limited resolution meant the enstrophy flux merely peaked and did not plateau. This implies the enstrophy flux found here has likely converged.

In the upper troposphere, upscale energy flux is due to two processes: eddy-eddy interactions source the synoptic-scale transient energy peak around $n=8$, and zonal mean-eddy interactions carry the energy from that scale up to planetary scales (Figs. 11 and 12). This is consistent with earlier findings of Shepherd (1987), but now the nonlinear interactions can be considered well resolved. Eddy-eddy interactions mainly transfer energy from around $n=15$ to around $n=8$, only about a factor of 2 in scale-the inverse cascade is limited, which is consistent with arguments that two-dimensional turbulence in the atmosphere plays a limited role because the atmosphere adjusts to a state of weak nonlinearity (Schneider and Walker 2006). On the other hand, the well-defined enstrophy flux mainly carried by eddy-eddy interactions down to the dissipation scale is consistent with two-dimensional turbulence theory.

That both upscale kinetic energy flux and downscale enstrophy flux maximize in the upper troposphere accords with wave-mean flow interaction theory (Held and Hoskins 1985). Eddy horizontal momentum fluxes peak at these altitudes and are the main mechanism for strengthening of the westerlies (kinetic energy transfer into the zonal mean flow). They arise from the horizontal component of the Eliassen-Palm flux, whose convergence relates to wave breaking in Rossby wave critical layers (Randel and Held 1991), which causes filamentation and downscale enstrophy transfer (McIntyre and Palmer 1985; Thorncroft et al. 1993).

We find no evidence for the balanced-upscale-cascade explanation of the shallow mesoscale spectrum (Lilly 1983; Vallis et al. 1997) seen in the ECMWF analyses above $250 \mathrm{hPa}$. Not only is the kinetic energy flux downscale in that range, the mesoscale spectrum is definitely not balanced, as divergent energy is not much smaller than rotational energy. In fact, the divergent contribution to the kinetic energy spectrum exceeds the rotational contribution in the mesoscale. Hence, the shallow mesoscale Nastrom-Gage-like spectrum does not reflect a two-dimensional turbulent inverse energy cascade, at least in the ECMWF IPY analysis.

In the stratosphere (100 hPa and higher), the energy source moves to planetary scales, and zonal mean-eddy interactions dominate the energy flux, which is largely confined to $n<10$. Zonal mean-eddy interactions also contribute half the downscale enstrophy flux, which should not be interpreted as homogeneous two-dimensional turbulence. The dominance of zonal mean-eddy transfers matches our understanding of wave-mean flow interaction in the stratosphere. Eddy-eddy interactions also play a significant role in downscale enstrophy flux, which is consistent with our understanding of Rossby wave breaking in the stratospheric surf zone (Juckes and McIntyre 1987). Notably, the downscale (rotational) energy flux at high wavenumbers in the stratosphere remains negligible (Fig. 8), even though there is a shallow spectrum of unbalanced kinetic energy at those scales. Because unbalanced flow includes a rotational component, this implies the unbalanced flow is not actively participating in nonlinear interactions or inducing a 
downscale energy flux of its own. It therefore presumably consists of upward-propagating internal gravity waves, as argued by Koshyk et al. (1999).

One limitation of the present study is restriction to global-mean spectra, made necessary by spherical harmonic analysis. It may be no coincidence that the transition layer found here, from 230 to $100 \mathrm{hPa}$, over which the spectral break moves rapidly to large scales, coincides roughly with the altitude range of the tropopause as one moves from high to low latitudes. Repeating this analysis with a localized spectral analysis technique, such as wavelets, would reveal whether the transition is sharper if examined in the extratropics alone.

Another limitation is the restriction to essentially barotropic diagnostics, such as kinetic energy spectra and horizontal spectral energy and enstrophy fluxes. Although this is reasonable within the context of geostrophic turbulence (Salmon 1980), examining baroclinic diagnostics is necessary to obtain a more complete description of the dynamics.

Acknowledgments. We are grateful to the SPARCIPY project for archiving the ECMWF IPY analysis, and to Nils Wedi of ECMWF for providing the recent T1279 forecast, as well as helpful comments on a draft. We also thank Mike Neish of the University of Toronto for technical support, Robert Field for an introduction to changepoint analysis, Richard Valent and Paul Swarztrauber at NCAR for assistance with Spherepack, and two anonymous reviewers for their comments. Funding was provided by the Natural Sciences and Engineering Research Council of Canada and the Canadian Foundation for Climate and Atmospheric Sciences. BHB was supported by an NSERC Canada Graduate Scholarship and an Amelia Earhart Fellowship from the Zonta International Foundation.

\section{APPENDIX}

\section{Decompositions of Kinetic Energy Spectrum}

The rotational and divergent kinetic energy spectra are, respectively,

$$
\begin{aligned}
& E_{n}^{\mathrm{rot}}=\frac{1}{4} \frac{n(n+1)}{a^{2}} \sum_{m=-n}^{n} \overline{\left|\psi_{n}^{m}\right|^{2}} \text { and } \\
& E_{n}^{\mathrm{div}}=\frac{1}{4} \frac{a^{2}}{n(n+1)} \sum_{m=-n}^{n} \overline{\left|\delta_{n}^{m}\right|^{2}} .
\end{aligned}
$$

We further decompose the fields into stationary and transient components. For the streamfunction, the decomposition is $\overline{\left|\psi_{n}^{m}\right|^{2}}=\left|\overline{\psi_{n}^{m}}\right|^{2}+\overline{\left|\psi_{n}^{m^{\prime}}\right|^{2}}$, where the overbar represents a monthly average, and the transient fluctuation around the monthly mean is $\psi_{n}^{m^{\prime}}=\psi_{n}^{m}-\overline{\psi_{n}^{m}}$. The total kinetic energy spectrum, decomposed into stationary and transient contributions, is then

$$
E_{n}=\frac{1}{4} \frac{a^{2}}{n(n+1)} \sum_{m=-n}^{n}\left(\left|\overline{\zeta_{n}^{m}}\right|^{2}+\left|\overline{\delta_{n}^{m}}\right|^{2}+\overline{\left|\zeta_{n}^{m^{\prime}}\right|^{2}}+\overline{\left|\delta_{n}^{m^{\prime}}\right|^{2}}\right)
$$

where $\zeta_{n}^{m}=-\left[n(n+1) / a^{2}\right] \psi_{n}^{m}$.

We also decompose the kinetic energy spectra into meridional and zonal components, labeled by $\varphi$ and $\lambda$, respectively. The formulas for the rotational flow are (Shepherd 1987)

$$
E_{\phi}^{\mathrm{rot}}(n)=\frac{1}{8 a^{2}} \sum_{m=-n}^{n}(2 n+1)|m| \overline{\left|\psi_{n}^{m}\right|^{2}}
$$

and

$$
E_{\lambda}^{\mathrm{rot}}(n)=\frac{1}{8 a^{2}} \sum_{m=-n}^{n}[2 n(n+1)-(2 n+1)|m|] \overline{\left.\psi_{n}^{m}\right|^{2}} .
$$

The corresponding decomposition for the divergent flow is

$E_{\phi}^{\operatorname{div}}(n)=\frac{a^{2}}{8} \sum_{m=-n}^{n} \frac{2 n(n+1)-(2 n+1)|m|}{n^{2}(n+1)^{2}} \mid \overline{\left.\delta_{n}^{m}\right|^{2}}$

and

$$
E_{\lambda}^{\operatorname{div}}(n)=\frac{a^{2}}{8} \sum_{m=-n}^{n} \frac{(2 n+1)|m|}{n^{2}(n+1)^{2}}\left|\delta_{n}^{m}\right|^{2}
$$

If the flow is homogeneous and isotropic, $\left|\psi_{n}^{m}\right|^{2}$ is independent of $m$ (Boer 1983). This holds for random fields in general, and therefore also for $\delta$. If $E_{\lambda}>E_{\phi}$, the flow is zonally anisotropic, which means the zonal motions dominate the meridional motions, whereas if $E_{\lambda}<E_{\phi}$, the flow is meridionally anisotropic, and the meridional motions dominate.

\section{REFERENCES}

Adams, J. C., and P. N. Swarztrauber, 1997: Spherepack 2.0: A model development facility. NCAR Tech. Note NCAR/ TN-436+STR, 62 pp.

Bacmeister, J., S. Eckermann, P. Newman, L. Lait, K. Chan, M. Loewenstein, M. Proffitt, and B. Gary, 1996: Stratospheric horizontal wavenumber spectra of winds, potential temperature, and atmospheric tracers observed by high-altitude aircraft. J. Geophys. Res., 101 (D5), 9441-9470.

Bartello, P., 2010: Quasigeostrophic and stratified turbulence in the atmosphere. Proc. IUTAM Symp. on Turbulence in the Atmosphere and Oceans, Cambridge, United Kingdom, IUTAM, 117-130. 
Boer, G. J., 1983: Homogeneous and isotropic turbulence on the sphere. J. Atmos. Sci., 40, 154-163.

— bulence in the atmosphere. J. Atmos. Sci., 40, 164-184.

Charney, J. G., 1971: Geostrophic turbulence. J. Atmos. Sci., 28, 1087-1095.

— , and P. G. Drazin, 1961: Propagation of planetary-scale disturbances from the lower into the upper atmosphere. J. Geophys. Res., 66, 83-109.

Cho, J. Y. N., and E. Lindborg, 2001: Horizontal velocity structure functions in the upper troposphere and lower stratosphere: 1. Observations. J. Geophys. Res., 106 (D10), 10 223-10 232.

Hamilton, K., Y. O. Takahashi, and W. Ohfuchi, 2008: Mesoscale spectrum of atmospheric motions investigated in a very fine resolution global general circulation model. J. Geophys. Res., 113, D18110, doi:10.1029/2008JD009785.

Held, I. M., and B. J. Hoskins, 1985: Large-scale eddies and the general circulation of the troposphere. Advances in Geophysics, Vol. 28, Academic Press, 3-31.

Hinkley, D. V., 1969: Inference about the intersection in two-phase regression. Biometrika, 56, 495-504.

Hirons, L. C., P. Inness, F. Vitart, and P. Bechtold, 2012: Understanding advances in the simulation of intraseasonal variability in the ECMWF model. Part II: The application of process-based diagnostics. ECMWF Tech. Memo. 682, 28 pp.

Juckes, M. N., and M. E. McIntyre, 1987: A high resolution, onelayer model of breaking planetary waves in the stratosphere. Nature, 328, 590-596.

Koshyk, J. N., and K. Hamilton, 2001: The horizontal kinetic energy spectrum and spectral budget simulated by a high-resolution troposphere-stratosphere-mesosphere GCM. J. Atmos. Sci., 58, 329-348.

— B. A. Boville, K. Hamilton, E. Manzini, and K. Shibata, 1999: Kinetic energy spectrum of horizontal motions in middleatmosphere models. J. Geophys. Res., 104 (D22), 27 17721190.

Kraichnan, R. H., 1967: Inertial ranges in two-dimensional turbulence. Phys. Fluids, 10,1417-1423.

Levenberg, K., 1944: A method for the solution of certain nonlinear problems in least squares. Quart. Appl. Math., 2, 164-168.

Lilly, D. K., 1983: Stratified turbulence and the mesoscale variability of the atmosphere. J. Atmos. Sci., 40, 749-761.

Lindborg, E., 2006: The energy cascade in a strongly stratified fluid. J. Fluid Mech., 550, 207-242.
McIntyre, M. E., and T. N. Palmer, 1985: A note on the general concept of wave breaking for Rossby and gravity waves. Pure Appl. Geophys., 123, 964-975.

Nastrom, G. D., and K. S. Gage, 1985: A climatology of atmospheric wavenumber spectra of wind and temperature observed by commercial aircraft. J. Atmos. Sci., 42, 950-960.

Palmer, T. N., 2001: A nonlinear dynamical perspective on model error: A proposal for non-local stochastic-dynamic parametrization in weather and climate prediction models. Quart. J. Roy. Meteor. Soc., 127, 279-304.

, G. J. Shutts, R. Hagedorn, F. J. Doblas-Reyes, T. Jung, and M. Leutbecher, 2005: Representing model uncertainty in weather and climate prediction. Annu. Rev. Earth Planet. Sci., 33, 163-193.

Pendlebury, D., 2009: SPARC-IPY data archive. SPARC Newsletter, No. 33, World Climate Research Programme, Toronto, Canada, 20.

Randel, W. J., and I. M. Held, 1991: Phase speed spectra of transient eddy fluxes and critical layer absorption. J. Atmos. Sci., 48, 688-697.

Salmon, R., 1980: Baroclinic instability and geostrophic turbulence. Geophys. Astrophys. Fluid Dyn., 12, 167-211.

Saujani, S., and T. G. Shepherd, 2006: A unified theory of balance in the extratropics. J. Fluid Mech., 569, 447-464.

Schneider, T., and C. C. Walker, 2006: Self-organization of atmospheric macroturbulence into critical states of weak nonlinear eddy-eddy interactions. J. Atmos. Sci., 63, 1569-1586.

Shepherd, T. G., 1987: A spectral view of nonlinear fluxes and stationary-transient interaction in the atmosphere. J. Atmos. Sci., 44, 1166-1178.

Straus, D. M., and P. Ditlevsen, 1999: Two-dimensional turbulence properties of the ECMWF reanalyses. Tellus, 51, 751-772.

Thorncroft, C. D., B. J. Hoskins, and M. E. McIntyre, 1993: Two paradigms of baroclinic-wave life-cycle behaviour. Quart. J. Roy. Meteor. Soc., 119, 17-55.

Toms, J. D., and M. L. Lesperance, 2003: Piecewise regression: A tool for identifying ecological thresholds. Ecology, 8, 2034-2041.

Trenberth, K. E., and A. Solomon, 1993: Implications of global atmospheric spectra for processing and displaying data. J. Climate, 6, 531-545.

Vallis, G. K., G. J. Shutts, and M. E. B. Grey, 1997: Balanced mesoscale motion and stratified turbulence forced by convection. Quart. J. Roy. Meteor. Soc., 123, 1621-1652.

Waite, M. L., and P. Bartello, 2004: Stratified turbulence dominated by vortical motion. J. Fluid Mech., 517, 281-308. 